\title{
Fixed points on Klein bottle fiber bundles over the circle
}

by

\author{
D. L. Gonçalves (São Paulo), D. Penteado (São Carlos) and \\ J. P. Vieira (Rio Claro)
}

\begin{abstract}
The main purpose of this work is to study fixed points of fiber-preserving maps over the circle $S^{1}$ for spaces which are fiber bundles over $S^{1}$ and the fiber is the Klein bottle $K$. We classify all such maps which can be deformed fiberwise to a fixed point free map. The similar problem for torus fiber bundles over $S^{1}$ has been solved recently.
\end{abstract}

Introduction. Given a fiber bundle $E \rightarrow B$ and a fiber-preserving map $f: E \rightarrow E$ over $B$, the question whether $f$ can be deformed over $B$ (by a fiberwise homotopy) to a fixed point free map has been considered by many authors (see for example [Do74], [FH81], [Go87]). In [FH81], Fadell and Husseini showed that the above problem can be stated in terms of obstructions (including higher ones). This was done under the hypothesis that the base space, the total space and the fiber $F$ are manifolds, and the dimension of $F$ is greater than or equal to 3 . The case where the fiber has dimension 2 was considered in [GPV04], where a few generalities were discussed and the fixed point problem over $B$ as defined above was completely solved for any torus fiber bundles over the circle $S^{1}$. In the present work we study the fixed point problem over $B$ for Klein bottle fiber bundles over $S^{1}$.

Recall that a Klein bottle bundle over $S^{1}$ has as total space the mapping torus $M(\phi)$ where $\phi: K \rightarrow K$ is a homeomorphism. A relevant step in solving the problem is to determine, for each fiber bundle $M(\phi) \rightarrow S^{1}$, the set of homotopy classes of maps $f$ over $S^{1}$ such that $f$ restricted to the fiber can be deformed to a fixed point free map. This is done in Theorem 2.4. The main result of the paper is Theorem 6.26, which gives a classification of the homotopy classes of fiber-preserving maps given by Theorem 2.4 which can be deformed over $S^{1}$ to a fixed point free map. Our method is to study solutions of a system of equations in a free group either by providing an explicit solution or by considering the system in some quotients of this group.

2000 Mathematics Subject Classification: Primary 55M20; Secondary 55R10, 55S35.

Key words and phrases: fixed point, fiber bundle, fiberwise homotopy, abelianized obstruction. 
The paper is organized in six sections. In Section 1 we give some results about the Klein bottle, $K$-bundles and an algebraic formulation of our main question. In Section 2 we classify all $K$-bundles over $S^{1}$ and bundle maps which have the property that their restriction to a fiber can be deformed to a fixed point free map (Theorem 2.4). In Section 3 we compute the fundamental group of the $K$-bundle $M(\phi) \times{ }_{S^{1}} M(\phi)$ with suitable base points. In Section 4 we compute the fundamental group of the $K$-bundle $M(\phi) \times_{S^{1}} M(\phi)-\Delta$ where $\Delta$ is the diagonal. In Section 5 we give necessary and sufficient conditions for the existence of a lifting in the algebraic diagram (1.1); this existence is equivalent to the map being fiberwise deformable to a fixed point free map (Theorem 5.1). Then we reduce the cases to be analyzed (Corollary 5.4). In Section 6 we derive necessary conditions for the relevant system of equations on a free group $F$ to have a solution, by looking at the system on some quotients of $F$. In certain cases we construct explicit solutions. Then we derive the main result, which is Theorem 6.26.

\section{Preliminaries}

1.1. The algebraic problem. Let $f: E \rightarrow E$ be a fiber-preserving map over $S^{1}$ where $E$ is a fiber bundle over $S^{1}$ with fiber a surface $S$. From Corollary 1.3 in [GPV04] we know that all such bundles are of the form $S \rightarrow M(\phi) \stackrel{p}{\rightarrow} S^{1}$ where $\phi: S \rightarrow S$ is a homeomorphism and $M(\phi)$ is the quotient of $S \times I$ by the relation $(x, 0) \sim(\phi(x), 1)$.

Let $M(\phi) \times{ }_{S^{1}} M(\phi)$ be the pullback of $p: M(\phi) \rightarrow S^{1}$ along $p: M(\phi)$ $\rightarrow S^{1}$, and $p_{i}: M(\phi) \times_{S^{1}} M(\phi) \rightarrow M(\phi), i=1,2$, the projections to the first and the second coordinates, respectively. The fixed point problem for a fiber-preserving map $f$ over $S^{1}$ is equivalent to the algebraic problem given by Proposition 1.6 from [GPV04]. So we must compute the groups and homomorphisms in the following diagram:

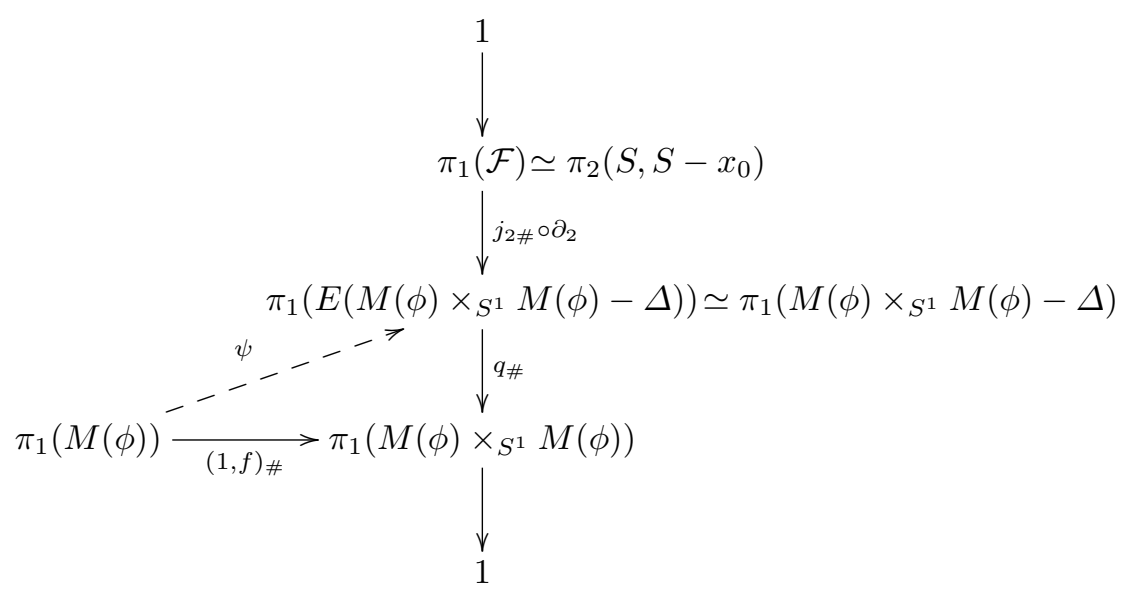


where $S$ is the Klein bottle and the homomorphisms are defined at the beginning of Section 5. The group $\pi_{2}\left(S, S-x_{0}\right)$ is well known and Propositions 1.7 and 1.8 of [GPV04] tell us how to compute the groups $\pi_{1}(M(\phi))$ and $\pi_{1}\left(M(\phi) \times{ }_{S^{1}} M(\phi)\right)$, respectively. To compute $\pi_{1}\left(M(\phi) \times_{S^{1}} M(\phi)-\Delta\right)$ we will use the proposition below, which holds for any closed surface $S$ different from $S^{2}$ and $\mathbb{R} P^{2}$.

Proposition 1.1. The fundamental group $\pi_{1}\left(M(\phi) \times_{S^{1}} M(\phi)-\Delta\right)$ is isomorphic to the semidirect product $\pi_{1}(S \times S-\Delta) \rtimes_{\theta} \mathbb{Z}$ for some action $\theta$.

Proof. The fiber bundle $S \times S-\Delta \rightarrow M(\phi) \times_{S^{1}} M(\phi)-\Delta \stackrel{p \circ p_{2 \mid}}{\longrightarrow} S^{1}$ provides the short exact sequence

$$
1 \rightarrow \pi_{1}(S \times S-\Delta) \rightarrow \pi_{1}\left(M(\phi) \times_{S^{1}} M(\phi)-\Delta\right) \stackrel{p \circ p_{2 \mid \#}}{\longrightarrow} \mathbb{Z} \rightarrow 1,
$$

and the result follows since $\mathbb{Z}$ is free.

1.2. Generators of $\pi_{1}(K)$ and the Nielsen number of a continuous map $f: K \rightarrow K$. Let $\pi_{1}(K)=\left\langle\alpha, \beta \mid \alpha \beta \alpha \beta^{-1}=1\right\rangle$ be the well known presentation of the fundamental group of the Klein bottle.

If $f: K \rightarrow K$ is a continuous map then $f_{\#}: \pi_{1}(K) \rightarrow \pi_{1}(K)$ is a homomorphism of the form:

- Type 1: $f_{\#}(\alpha)=1, f_{\#}(\beta)=\alpha^{p} \beta^{2 q}$,

- Type 2: $f_{\#}(\alpha)=\alpha^{r}, f_{\#}(\beta)=\alpha^{p} \beta^{2 q+1}$.

In some situations we will distinguish the two types, but not always.

To compute the Nielsen number of a map $f: K \rightarrow K$ given by $f_{\#}(\alpha)$ $=\alpha^{r}, f_{\#}(\beta)=\alpha^{s} \beta^{t}$ we have the following result of [DJ93].

TheOrem 1.2. The Nielsen number of the above map $f: K \rightarrow K$ is

$$
N(f)=|t-1| \max \{1,|r|\} .
$$

Corollary 1.3. If $f: K \rightarrow K$ is a continuous map so that $f_{\#}$ : $\pi_{1}(K) \rightarrow \pi_{1}(K)$ is of type 2 , then

$$
N(f)= \begin{cases}|2 r q| & \text { if } r \neq 0, \\ |2 q| & \text { if } r=0 .\end{cases}
$$

2. The Klein bottle case-preliminary reductions. We use some homeomorphisms of the Klein bottle to describe all $K$-bundles over $S^{1}$ up to fiberwise isotopy.

Let us consider in $\mathbb{R}^{2}$ the equivalence relation generated by the relations $(x, y) \sim(x, y+1)$ and $(x, y) \sim(x+1,1-y)$. The quotient space is $K$ and the equivalence class of $(x, y) \in \mathbb{R}^{2}$ is denoted by $[(x, y)] \in K$.

Let $\phi$ be a homeomorphism of $K$ so that $\phi([(0,0)])=[(0,0)]=x_{2}$.

As in Section 1 , let $M(\phi)$ be the quotient space of $K \times[0,1]$, where we identify $([(x, y)], 0)$ with $(\phi[(x, y)], 1)$. The class of $([(x, y)], t)$ in the quotient 
is denoted by $\langle[(x, y)], t\rangle$. The space $M(\phi)$ is a fiber bundle over the circle $S^{1}$, where the fiber is the Klein bottle. The projection map $p: M(\phi) \rightarrow S^{1}$ is given by $p(\langle[(x, y)], t\rangle)=\langle t\rangle \in[0,1] / 0 \simeq 1 \simeq S^{1}$.

We denote by $f_{s}(r, t): \alpha \mapsto \alpha^{r}, \beta \mapsto \alpha^{s} \beta^{t}$ the homomorphism on $\pi_{1}(K)$ induced by the restriction of $f$ to $K$, and by $\phi_{p}(\epsilon, \eta): \alpha \mapsto \alpha^{\epsilon}, \beta \mapsto \alpha^{p} \beta^{\eta}$ the isomorphism of $\pi_{1}(K)$ induced by the homeomorphism $\phi$. Since $\phi_{p}(\epsilon, \eta)$ is an isomorphism it follows that $\epsilon= \pm 1$ and $\eta= \pm 1$.

Proposition 2.1.

(1) $\pi_{1}\left(M\left(\phi_{p}(\epsilon, \eta)\right), \mathbf{0}\right)=\left\langle\alpha, \beta, c_{0}\right| \alpha \beta \alpha \beta^{-1}=1, c_{0} \alpha c_{0}{ }^{-1}=\alpha^{\epsilon}, c_{0} \beta c_{0}{ }^{-1}$ $\left.=\alpha^{p} \beta^{\eta}\right\rangle$, where $\mathbf{0}=\langle[(0,0)], 0\rangle$.

(2) There are four isotopy classes of homeomorphisms of $K$ where a set of representatives is given by $\left\{\phi_{0}(1,1), \phi_{1}(1,1), \phi_{0}(1,-1), \phi_{1}(1,-1)\right\}$.

(3) For any homeomorphism $\phi: K \rightarrow K, M(\phi)$ is homeomorphic over $S^{1}$ to $M\left(\phi_{p}(1, \eta)\right)$ where $\phi_{p}(1, \eta)$ is given by (1). Further, the $M\left(\phi_{p}(1, \eta)\right)$ 's are not homeomorphic for two different $\phi_{p}(1, \eta)$ given in $(2)$.

Proof. (1) This follows from Proposition 1.7 in [GPV04].

(2) It is a straightforward calculation to show that the number of conjugacy classes of isomorphisms of $\pi_{1}(K)$ is four and a set of representatives is given by $\left\{\phi_{0}(1,1), \phi_{1}(1,1), \phi_{0}(1,-1), \phi_{1}(1,-1)\right\}$. Since the surface $K$ is a $K(\pi, 1)$ we can identify these classes with the isotopy classes of $K$.

(3) The first part follows from Proposition 1.2 in [GPV04]. The last part follows from the fact that the spaces have nonisomorphic fundamental groups (given before the proposition).

Proposition 2.2. If $f$ is deformable to a fixed point free map over $S^{1}$ then the Nielsen number of $f$ restricted to the fiber is zero, which implies that $f_{s}(r, t)$ is of the form $f_{s}(r, 1)$.

Proof. The first part is clear. That $f_{s}(r, t)$ is of the form $f_{s}(r, 1)$ follows from Corollary 1.3.

Let us denote by $f_{s}\left(r, t, c_{1}, c_{2}\right): \pi_{1}\left(M\left(\phi_{q}(1, \eta)\right)\right) \rightarrow \pi_{1}\left(M\left(\phi_{q}(1, \eta)\right)\right)$ the homomorphism $\alpha \mapsto \alpha^{r}, \beta \mapsto \alpha^{s} \beta^{t}$ and $c_{0} \mapsto \alpha^{c_{1}} \beta^{c_{2}} c_{0}$. Consequently, $r, s, t, c_{1}, c_{2}$ must satisfy certain equations as a result of the relations in the group.

Proposition 2.3. Let $f: M\left(\phi_{q}(1, \eta)\right) \rightarrow M\left(\phi_{q}(1, \eta)\right)$ be a map over $S^{1}$, where $q \in\{0,1\}$ and $\eta= \pm 1$. If the Nielsen number of $f$ restricted to the fiber is zero then $f_{\#}: \pi_{1}\left(M\left(\phi_{q}(1, \eta)\right)\right) \rightarrow \pi_{1}\left(M\left(\phi_{q}(1, \eta)\right)\right)$ has the form $f_{s}\left(r, 1, c_{1}, c_{2}\right)$ where

(i) $(-1)^{c_{2}} r=r$,

(ii) $2 c_{1}=s\left[1-(-1)^{c_{2}}\right]+q\left[r-(-1)^{c_{2}}\right]$. 
Conversely, for each homomorphism $f_{s}\left(r, 1, c_{1}, c_{2}\right): \pi_{1}\left(M\left(\phi_{q}(1, \eta)\right)\right) \rightarrow$ $\pi_{1}\left(M\left(\phi_{q}(1, \eta)\right)\right)$, with $q \in\{0,1\}$ and $\eta= \pm 1$, where $\left(r, s, c_{1}, c_{2}, q\right)$ satisfy the conditions (i) and (ii) above, there is a map $f: M\left(\phi_{q}(1, \eta)\right) \rightarrow M\left(\phi_{q}(1, \eta)\right)$ over $S^{1}$ such that $f_{\#}=f_{s}\left(r, 1, c_{1}, c_{2}\right)$ and the Nielsen number of $f$ restricted to the fiber is zero.

Proof. Since $f: M\left(\phi_{q}(1, \eta)\right) \rightarrow M\left(\phi_{q}(1, \eta)\right)$ is a map over $S^{1}$ we have the following commutative diagram:

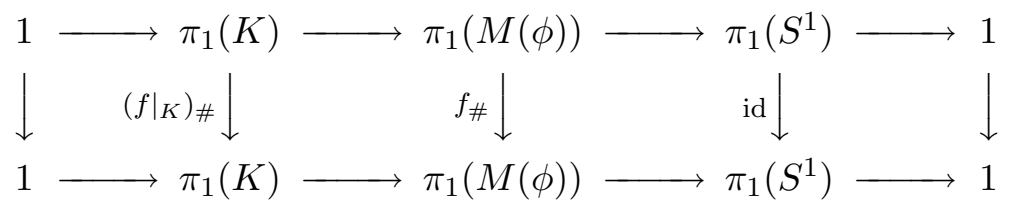

Because $\left.f\right|_{K}$ has Nielsen number zero, $\left(\left.f\right|_{K}\right)_{\#}=f_{s}(r, 1)$ for some $r, s \in \mathbb{Z}$. Therefore $f_{\#}=f_{s}\left(r, 1, c_{1}, c_{2}\right)$.

Now we will show (i) and (ii).

Because $\pi_{1}\left(M\left(\phi_{q}(1, \eta)\right)\right)=\left\langle\alpha, \beta, c_{0}\right| \alpha \beta \alpha \beta^{-1}=1, c_{0} \alpha c_{0}^{-1}=\alpha, c_{0} \beta c_{0}^{-1}=$ $\left.\alpha^{q} \beta^{\eta}\right\rangle$ by Proposition 2.1 , we must have $f_{\#}\left(c_{0} \alpha c_{0}^{-1}\right)=f_{\#}(\alpha)$ and $f_{\#}\left(c_{0} \beta c_{0}^{-1}\right)$ $=f_{\#}\left(\alpha^{q} \beta^{\eta}\right)$. Using

$$
\left(\alpha^{r} \beta^{s}\right)^{t}= \begin{cases}\alpha^{\frac{t}{2}\left[r\left(1+(-1)^{s}\right)\right]} \beta^{s t} & \text { if } t \text { is even, } \\ \alpha^{\frac{t-1}{2}\left[r\left(1+(-1)^{s}\right)\right]+r} \beta^{s t} & \text { if } t \text { is odd, }\end{cases}
$$

we have $(-1)^{c_{2}} r=r$ and $2 c_{1}=s\left[1-(-1)^{c_{2}}\right]+q\left[r-(-1)^{c_{2}}\right]$. This finishes the proof of the first part.

For the converse we first observe that $p_{\#} \circ f_{s}\left(r, 1, c_{1}, c_{2}\right)=p_{\#}$. Because all spaces are $K(\pi, 1)$ there exists $g: M\left(\phi_{q}(1, \eta)\right) \rightarrow M\left(\phi_{q}(1, \eta)\right)$ and a homotopy $\left.H: M\left(\phi_{q}(1, \eta)\right) \times I, x_{1} \times I\right) \rightarrow\left(S^{1}, 1\right)$ so that $H(x, 0)=p$ 。 $g(x)$ and $H(x, 1)=p(x)$ and $g_{\#}=f_{s}\left(r, 1, c_{1}, c_{2}\right)$. The map $G:\left(x_{1} \times I \cup\right.$ $\left.M\left(\phi_{q}(1, \eta)\right) \times 0, x_{1} \times I\right) \rightarrow\left(M\left(\phi_{q}(1, \eta)\right), x_{2}\right)$ defined by $G(x, 0)=g(x)$ and $G\left(x_{1} \times I\right)=x_{2}$ makes the diagram

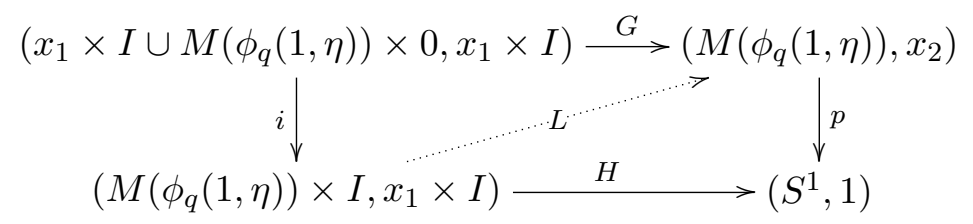

commutative. Since $p:\left(M\left(\phi_{q}(1, \eta)\right), x_{2}\right) \rightarrow\left(S^{1}, 1\right)$ is a fiber bundle it follows that there exists $L:\left(M\left(\phi_{q}(1, \eta)\right) \times I, x_{1} \times I\right) \rightarrow\left(M\left(\phi_{q}(1, \eta)\right), x_{2}\right)$ such that $p \circ L=H$ and $f=L(, 1):\left(M\left(\phi_{q}(1, \eta)\right), x_{1}\right) \rightarrow\left(M\left(\phi_{q}(1, \eta)\right), x_{2}\right)$ is over $S^{1}$, and the induced homomorphism on the fundamental group is $f_{s}\left(r, 1, c_{1}, c_{2}\right)$; indeed, $p \circ f=p \circ L(, 1)=H(, 1)=p$ and $f_{\#}=L(, 0)_{\#}=$ $g_{\#}=f_{s}\left(r, 1, c_{1}, c_{2}\right)$.

Then we have 
Theorem 2.4. Let $f: M\left(\phi_{q}(1, \eta)\right) \rightarrow M\left(\phi_{q}(1, \eta)\right)$ be a map over $S^{1}$, where $q \in\{0,1\}$ and $\eta= \pm 1$. If the Nielsen number of $f$ restricted to the fiber is zero then $f_{\#}: \pi_{1}\left(M\left(\phi_{q}(1, \eta)\right)\right) \rightarrow \pi_{1}\left(M\left(\phi_{q}(1, \eta)\right)\right)$ is given by the table:

\begin{tabular}{|l|lrll|}
\hline Case I & I.1: & $f_{s}(r, 1,0,2 k): \alpha \mapsto \alpha^{r}, \beta \mapsto \alpha^{s} \beta, c_{0} \mapsto \beta^{2 k} c_{0}$ & $r, s, k \in \mathbb{Z}$ \\
$\phi_{0}(1,1)$ & I.2: & $f_{s}(0,1, s, 2 k+1): \alpha \mapsto 1, \beta \mapsto \alpha^{s} \beta, c_{0} \mapsto \alpha^{s} \beta^{2 k+1} c_{0}$ & $s, k \in \mathbb{Z}$ \\
\hline Case II & & $f_{s}(2 r+1,1, r, 2 k): \alpha \mapsto \alpha^{2 r+1}, \beta \mapsto \alpha^{s} \beta, c_{0} \mapsto \alpha^{r} \beta^{2 k} c_{0}$ & $r, s, k \in \mathbb{Z}$ \\
$\phi_{1}(1,1)$ & & & \\
\hline Case III & III.1: & $f_{s}(r, 1,0,2 k): \alpha \mapsto \alpha^{r}, \beta \mapsto \alpha^{s} \beta, c_{0} \mapsto \beta^{2 k} c_{0}$ & $r, s, k \in \mathbb{Z}$ \\
$\phi_{0}(1,-1)$ & III.2: & $f_{s}(0,1, s, 2 k+1): \alpha \mapsto 1, \beta \mapsto \alpha^{s} \beta, c_{0} \mapsto \alpha^{s} \beta^{2 k+1} c_{0}$ & $s, k \in \mathbb{Z}$ \\
\hline Case IV & & $f_{s}(2 r+1,1, r, 2 k): \alpha \mapsto \alpha^{2 r+1}, \beta \mapsto \alpha^{s} \beta, c_{0} \mapsto \alpha^{r} \beta^{2 k} c_{0}$ & $r, s, k \in \mathbb{Z}$ \\
$\phi_{1}(1,-1)$ & & & \\
\hline
\end{tabular}

Proof. By Proposition 2.3, we have $2 c_{1}=s\left[1-(-1)^{c_{2}}\right]+q\left[r-(-1)^{c_{2}}\right]$ and $(-1)^{c_{2}} r=r$. Using these equations for various values of $\eta \in\{1,-1\}$ and $q \in\{0,1\}$ yields the result.

3. Calculation of $\pi_{1}\left(M(\phi) \times{ }_{S^{1}} M(\phi),\left(\left\langle x_{2}, 0\right\rangle,\left\langle x_{i}, 0\right\rangle\right)\right), i=1,2$. Recall that $x_{2}=[(0,0)]$ and set $x_{1}=[(0, q)]$ for $q$ small. Later we will choose $\phi$ such that also $\phi\left(x_{1}\right)=x_{1}$. We have the short exact sequence

$1 \rightarrow \pi_{1}\left(K, x_{i}\right) \stackrel{l_{\#}}{\longrightarrow} \pi_{1}\left(M(\phi) \times_{S^{1}} M(\phi),\left(\left\langle x_{2}, 0\right\rangle,\left\langle x_{i}, 0\right\rangle\right)\right) \stackrel{\left(p_{1}\right)_{\#}}{\longrightarrow} \pi_{1}\left(M(\phi),\left\langle x_{2}, 0\right\rangle\right) \rightarrow 1$

where $l_{\#}$ is the homomorphism induced by the map $l: K \rightarrow M(\phi) \times_{S^{1}} M(\phi)$ given by $x \mapsto\left(\left\langle x_{2}, 0\right\rangle,\langle x, 0\rangle\right)$, and $\left(p_{1}\right)_{\#}$ is induced by the map $p_{1}: M(\phi) \times_{S^{1}}$ $M(\phi) \rightarrow M(\phi)$ given by $(\langle x, t\rangle,\langle y, t\rangle) \mapsto\langle x, t\rangle$.

We will define an explicit set of generators for $\pi_{1}(K)$. For this we start by choosing a set of elements of $\pi_{1}(K-x)$ which will also be used in the next section. Let $\varrho_{11}, \varrho_{12}, B_{12}$ and $\varrho_{21}, \varrho_{22}$ be the elements of $\pi_{1}\left(K-x_{2}, x_{1}\right)$ and $\pi_{1}\left(K-x_{1}, x_{2}\right)$, as defined in [S69], respectively. See Figures 1 and 2.

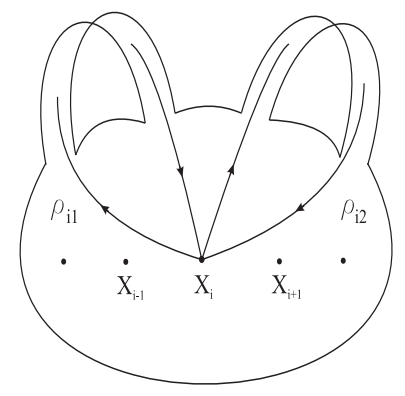

Fig. 1. The braids $\varrho_{i 1}$ and $\varrho_{i 1}$

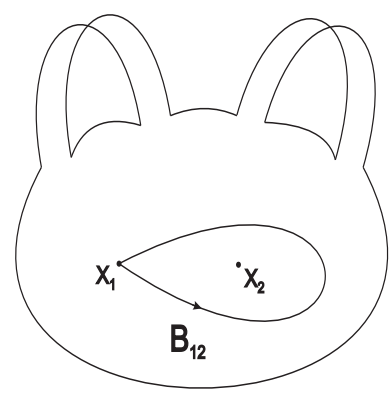

Fig. 2. The braid $B_{12}$ 
By abuse of notation denote also by $\varrho_{i j}$ the element of $\pi_{1}(K)$ which is the image of the $\varrho_{i j}$ defined above under the map induced by the inclusion $K-x \hookrightarrow K$.

We consider the presentations $\pi_{1}\left(K, x_{i}\right)=\left\langle a_{i}, b_{i} \mid a_{i} b_{i} a_{i} b_{i}^{-1}=1\right\rangle$ where $a_{i}=\varrho_{i 1} \varrho_{i 2}$ and $b_{i}=\varrho_{i 2}^{-1}$ and $\pi_{1}\left(M(\phi),\left\langle x_{2}, 0\right\rangle\right)=\left\langle\alpha, \beta, c_{0}\right| \alpha \beta \alpha \beta^{-1}=1$, $\left.\left.c_{0} \alpha c_{0}^{-1}=\alpha, c_{0} \beta c_{0}^{-1}=\alpha^{p} \beta^{\eta}\right)\right\rangle$ where $\eta= \pm 1$ and $p \in\{0,1\}$.

Denote by $\alpha_{1}, \beta_{1}, c_{01}, u_{1}, v_{1}$ the homotopy classes of the loops given respectively by the pairs of loops $\left(\alpha(t),\left\langle x_{1}, 0\right\rangle\right),\left(\beta(t),\left\langle x_{1}, 0\right\rangle\right),\left(c_{0}(t),\left\langle x_{1}, t\right\rangle\right)$, $\left(\left\langle x_{2}, 0\right\rangle, a_{1}(t)\right),\left(\left\langle x_{2}, 0\right\rangle, b_{1}(t)\right)$; and $\alpha_{2}, \beta_{2}, c_{02}, u_{2}, v_{2}$ the homotopy classes of the loops given respectively by the pairs of loops $\left(\alpha(t),\left\langle x_{2}, 0\right\rangle\right),\left(\beta(t),\left\langle x_{2}, 0\right\rangle\right)$, $\left(c_{0}(t), c_{0}(t)\right),\left(\left\langle x_{2}, 0\right\rangle, \alpha(t)\right),\left(\left\langle x_{2}, 0\right\rangle, \beta(t)\right)$.

TheOREm 3.1. Let $\phi_{p}(1, \eta)$ be one of the four cases given by Theorem 2.4 and $\alpha_{i}, \beta_{i}, c_{0 i}, u_{i}, v_{i}$ the elements in $\pi_{1}\left(M(\phi) \times_{S^{1}} M(\phi),\left(\left\langle x_{2}, 0\right\rangle,\left\langle x_{i}, 0\right\rangle\right)\right)$. Then $\pi_{1}\left(M(\phi) \times_{S^{1}} M(\phi),\left(\left\langle x_{2}, 0\right\rangle,\left\langle x_{i}, 0\right\rangle\right)\right)=\left\langle\alpha_{i}, \beta_{i}, c_{0 i}, u_{i}, v_{i}\right| u_{i} v_{i} u_{i} v_{i}^{-1}=1$, $\alpha_{i} \beta_{i} \alpha_{i} \beta_{i}^{-1}=1, c_{0 i} \alpha_{i} c_{0 i}^{-1} \alpha_{i}^{-1}=1, c_{0 i} \beta_{i} c_{0 i}{ }^{-1} \beta_{i}^{-\eta} \alpha_{i}^{-p}=1, \alpha_{i} u_{i} \alpha_{i}^{-1}=u_{i}$, $\left.\alpha_{i} v_{i} \alpha_{i}^{-1}=v_{i}, \beta_{i} u_{i} \beta_{i}^{-1}=u_{i}, \beta_{i} v_{i} \beta_{i}^{-1}=v_{i}, c_{0 i} u_{i} c_{0 i}^{-1}=u_{i}, c_{0 i} v_{i} c_{0 i}^{-1}=u_{i}^{p} v_{i}^{\eta}\right\rangle$.

Proof. The result follows promptly from Proposition 1.8 of [GPV04]. Also it is a corollary of Theorem 4.1 by letting $B$ be the trivial element in the presentation given by that theorem.

4. Calculation of $\pi_{1}\left(M(\phi) \times_{S^{1}} M(\phi)-\Delta,\left(\left\langle x_{2}, 0\right\rangle,\left\langle x_{1}, 0\right\rangle\right)\right)$. As before we have $x_{2}=[(0,0)], x_{1}=[(0, q)]$, and $\phi: K \rightarrow K$ is a homeomorphism such that $\phi\left(x_{2}\right)=x_{2}$ and $\phi\left(x_{1}\right)=x_{1}$.

In order to compute $\pi_{1}\left(M(\phi) \times_{S^{1}} M(\phi)-\Delta,\left(\left\langle x_{2}, 0\right\rangle,\left\langle x_{1}, 0\right\rangle\right)\right)$ we consider the fiber bundle

$$
\left(K-x_{2}, x_{1}\right) \stackrel{j_{2}}{\rightarrow}\left(M(\phi) \times_{S^{1}} M(\phi)-\Delta,\left(\left\langle x_{2}, 0\right\rangle,\left\langle x_{1}, 0\right\rangle\right)\right) \stackrel{p_{1 \mid}}{\longrightarrow}\left(M(\phi),\left\langle x_{2}, 0\right\rangle\right)
$$

where $j_{2}: K-x_{2} \rightarrow M(\phi) \times_{S^{1}} M(\phi)-\Delta$ is given by $y \mapsto\left(\left\langle x_{2}, 0\right\rangle,\langle y, 0\rangle\right)$ and $p_{1 \mid}: M(\phi) \times_{S^{1}} M(\phi)-\Delta \rightarrow M(\phi)$ is given by $(\langle x, t\rangle,\langle y, t\rangle)$ (with $\left.y \neq x\right)$ $\mapsto\langle x, t\rangle$. The homotopy sequence of this bundle is

$$
1 \rightarrow \pi_{1}\left(K-x_{2}, x_{1}\right) \stackrel{j_{2 \#}}{\longrightarrow} \pi_{1}\left(M(\phi) \times_{S^{1}} M(\phi)-\Delta,\left(\left\langle x_{2}, 0\right\rangle,\left\langle x_{1}, 0\right\rangle\right)\right) \stackrel{\left(p_{1} \mid\right)_{\#}}{\longrightarrow} \pi_{1}\left(M(\phi),\left\langle x_{2}, 0\right\rangle\right) \rightarrow 1 .
$$

We consider the presentations $\pi_{1}\left(K-x_{2}, x_{1}\right)=\langle\bar{a}, \bar{b}\rangle$ (the free group on the set $\{\bar{a}, \bar{b}\})$ where $\bar{a}=\varrho_{11} \varrho_{12}$ and $\bar{b}=\varrho_{12}^{-1}$, and $\pi_{1}\left(M(\phi),\left\langle x_{2}, 0\right\rangle\right)=\left\langle\alpha, \beta, c_{0}\right|$ $\left.\alpha \beta \alpha \beta^{-1}=1, c_{0} \alpha c_{0}^{-1}=\alpha, c_{0} \beta c_{0}^{-1}=\alpha^{p} \beta^{\eta}\right\rangle$ where $\eta= \pm 1, p \in\{0,1\}$. The $\varrho_{i j}$ were defined in the previous section, and $\alpha, \beta, c_{0}: I \rightarrow M(\phi)$ are given by $\alpha=\left\langle\varrho_{21} \varrho_{22}, 0\right\rangle, \beta=\left\langle\varrho_{22}^{-1}, 0\right\rangle$ and $c_{0}=\left\langle x_{2}, t\right\rangle$. Finally, consider the elements $\tilde{\alpha}, \tilde{\beta}, \tilde{c}_{0}, \tilde{a}, \tilde{b}: I \rightarrow M(\phi) \times_{S^{1}} M(\phi)-\Delta$ given by $\tilde{\alpha}=\left(\alpha,\left\langle x_{1}, 0\right\rangle\right)$, $\tilde{\beta}=\left(\beta,\left\langle x_{1}, 0\right\rangle\right), \tilde{c}_{0}=\left(c_{0},\left\langle x_{1}, t\right\rangle\right), \tilde{a}=\left(\left\langle x_{2}, 0\right\rangle,\langle\bar{a}, 0\rangle\right)$ and $\tilde{b}=\left(\left\langle x_{2}, 0\right\rangle,\langle\bar{b}, 0\rangle\right)$.

According to [Jo76, Chap. 13, Theorem 1], there is a presentation 


$$
\begin{array}{r}
\pi_{1}\left(M(\phi) \times{ }_{S^{1}} M(\phi)-\Delta,\left(\left\langle x_{2}, 0\right\rangle,\left\langle x_{1}, 0\right\rangle\right)\right)=\left\langle\tilde{\alpha}, \tilde{\beta}, \tilde{c}_{0}, \tilde{a}, \tilde{b}\right| \tilde{\alpha} \tilde{\beta} \tilde{\alpha} \tilde{\beta}^{-1}=p_{1}(\tilde{a}, \tilde{b}) \\
\tilde{c}_{0} \tilde{\alpha} \tilde{c}_{0}^{-1} \tilde{\alpha}^{-\epsilon}=p_{2}(\tilde{a}, \tilde{b}), \tilde{c}_{0} \tilde{\beta} \tilde{c}_{0}^{-1} \tilde{\beta}^{-\eta} \tilde{\alpha}^{-p}=p_{3}(\tilde{a}, \tilde{b}), \tilde{\alpha} \tilde{a} \tilde{\alpha}^{-1}=p_{4}(\tilde{a}, \tilde{b}) \\
\tilde{\alpha} \tilde{b} \tilde{\alpha}^{-1}=p_{5}(\tilde{a}, \tilde{b}), \tilde{\beta} \tilde{a} \tilde{\beta}^{-1}=p_{6}(\tilde{a}, \tilde{b}), \tilde{\beta} \tilde{b} \tilde{\beta}^{-1}=p_{7}(\tilde{a}, \tilde{b}), \tilde{c}_{0} \tilde{a} \tilde{c}_{0}^{-1}=p_{8}(\tilde{a}, \tilde{b}) \\
\left.\tilde{c}_{0} \tilde{b} \tilde{c}_{0}^{-1}=p_{9}(\tilde{a}, \tilde{b})\right\rangle
\end{array}
$$

where $p_{j}(\tilde{a}, \tilde{b}), j=1, \ldots, 9$, are words in $\tilde{a}$ and $\tilde{b}$.

Theorem 4.1. Let $\phi_{p}(1, \eta)$ be one of the four cases given by Theorem 2.4 and $\tilde{\alpha}, \tilde{\beta}, \tilde{c}_{0}, \tilde{a}, \tilde{b}$ the elements in $\pi_{1}\left(M(\phi) \times{ }_{S^{1}} M(\phi)-\Delta,\left(\left\langle x_{2}, 0\right\rangle,\left\langle x_{1}, 0\right\rangle\right)\right)$ defined above. Then

$$
\begin{aligned}
& \pi_{1}\left(M(\phi) \times_{S^{1}} M(\phi)-\Delta,\left(\left\langle x_{2}, 0\right\rangle,\left\langle x_{1}, 0\right\rangle\right)\right) \\
& =\left\langle\tilde{\alpha}, \tilde{\beta}, \tilde{c}_{0}, \tilde{a}, \tilde{b}\right| \tilde{\alpha} \tilde{\beta} \tilde{\alpha} \tilde{\beta}^{-1}=p_{1}(\tilde{a}, \tilde{b}), \tilde{c}_{0} \tilde{\alpha} \tilde{c}_{0}^{-1} \tilde{\alpha}^{-1}=p_{2}(\tilde{a}, \tilde{b}), \\
& \quad \tilde{c}_{0} \tilde{\beta} \tilde{c}_{0}^{-1} \tilde{\beta}^{-\eta} \tilde{\alpha}^{-p}=p_{3}(\tilde{a}, \tilde{b}), \tilde{\alpha} \tilde{a} \tilde{\alpha}^{-1}=p_{4}(\tilde{a}, \tilde{b}), \tilde{\alpha} \tilde{b} \tilde{\alpha}^{-1}=p_{5}(\tilde{a}, \tilde{b}), \\
& \left.\tilde{\beta} \tilde{a} \tilde{\beta}^{-1}=p_{6}(\tilde{a}, \tilde{b}), \tilde{\beta} \tilde{b} \tilde{\beta}^{-1}=p_{7}(\tilde{a}, \tilde{b}), \tilde{c}_{0} \tilde{a} \tilde{c}_{0}^{-1}=p_{8}(\tilde{a}, \tilde{b}), \tilde{c}_{0} \tilde{b} \tilde{c}_{0}^{-1}=p_{9}(\tilde{a}, \tilde{b})\right\rangle
\end{aligned}
$$

where $p_{j}(\tilde{a}, \tilde{b}), j=1, \ldots, 9$, are words in $\tilde{a}$ and $\tilde{b}$ given by the tables

$$
\begin{array}{|c|c|}
\hline \tilde{\alpha} \tilde{\beta} \tilde{\alpha} \tilde{\beta}^{-1}=B=\tilde{a} \tilde{b} \tilde{a} \tilde{b}^{-1} & \\
\hline \tilde{\alpha} \tilde{a} \tilde{\alpha}^{-1}=B \tilde{a} B^{-1} & \tilde{\beta} \tilde{a} \tilde{\beta}^{-1}=\tilde{b}^{-1} \tilde{a}^{-1} \tilde{b} \\
\hline \tilde{\alpha} \tilde{b} \tilde{\alpha}^{-1}=B\left(\tilde{a}^{-1} \tilde{b} \tilde{a}^{-1}\right) B^{-1} & \tilde{\beta} \tilde{b} \tilde{\beta}^{-1}=\tilde{b}^{-1}(B \tilde{b}) \tilde{b} \\
\hline
\end{array}
$$

and

\begin{tabular}{|l|rc|}
\hline Case I & $\tilde{c}_{0} \tilde{\alpha} \tilde{c}_{0}^{-1}=\tilde{\alpha}$ & $\tilde{c}_{0} \tilde{\beta} \tilde{c}_{0}^{-1}=\tilde{\beta}$ \\
$\phi_{0}(1,1)$ & $\tilde{c}_{0} \tilde{a} \tilde{c}_{0}^{-1}=\tilde{a}$ & $\tilde{c}_{0} \tilde{b} \tilde{c}_{0}^{-1}=\tilde{b}$ \\
\hline Case II & $\tilde{c}_{0} \tilde{\alpha} \tilde{c}_{0}^{-1}=\tilde{\alpha}$ & $\tilde{c}_{0} \tilde{\beta} \tilde{c}_{0}^{-1}=B^{-1} \tilde{\alpha} \tilde{\beta}$ \\
$\phi_{1}(1,1)$ & $\tilde{c}_{0} \tilde{a} \tilde{c}_{0}^{-1}=\tilde{a}$ & $\tilde{c}_{0} \tilde{b} \tilde{c}_{0}^{-1}=\tilde{b} \tilde{a}^{-1}$ \\
\hline Case III & $\tilde{c}_{0} \tilde{\alpha} \tilde{c}_{0}^{-1}=B^{-1} \tilde{\alpha}$ & $\tilde{c}_{0} \tilde{\beta} \tilde{c}_{0}^{-1}=\tilde{\beta} \tilde{\beta}^{-1}$ \\
$\phi_{0}(1,-1)$ & $\tilde{c}_{0} \tilde{a} \tilde{c}_{0}^{-1}=\tilde{a} B^{-1}$ & $\tilde{c}_{0} \tilde{b} \tilde{c}_{0}^{-1}=B \tilde{b}^{-1} B^{-1}$ \\
\hline Case IV & $\tilde{c}_{0} \tilde{\alpha} \tilde{c}_{0}^{-1}=B^{-1} \tilde{\alpha}$ & $\tilde{c}_{0} \tilde{\beta} \tilde{c}_{0}^{-1}=\tilde{\alpha} \tilde{\beta}^{-1}$ \\
$\phi_{1}(1,-1)$ & $\tilde{c}_{0} \tilde{a} \tilde{c}_{0}^{-1}=\tilde{a} B^{-1}$ & $\tilde{c}_{0} \tilde{b} \tilde{c}_{0}^{-1}=B \tilde{b}^{-1} \tilde{a}^{-1}$ \\
\hline
\end{tabular}

Proof. To simplify we set $\bar{\alpha}=\varrho_{21} \varrho_{22}$ and $\bar{\beta}=\varrho_{22}^{-1}$. Since $\left(\bar{\alpha} \bar{\beta} \bar{\alpha} \bar{\beta}^{-1}, x_{1}\right)$ $=\left(\varrho_{21} \varrho_{22} \varrho_{22}^{-1} \varrho_{21} \varrho_{22} \varrho_{22}, x_{1}\right)=\left(\varrho_{21}^{2} \varrho_{22}^{2}, x_{1}\right)$ is homotopic to $\left(x_{2}, \varrho_{11}^{2} \varrho_{12}^{2}\right)=$ $\left(x_{2}, \bar{a} \bar{b} \bar{a} \bar{b}^{-1}\right)$ it follows that

$$
\begin{aligned}
\tilde{\alpha} \tilde{\beta} \tilde{\alpha} \tilde{\beta}^{-1} & =\left(\alpha \beta \alpha \beta^{-1},\left\langle x_{1}, 0\right\rangle\right)=\left(\left\langle\bar{\alpha} \bar{\beta} \bar{\alpha} \bar{\beta}^{-1}, 0\right\rangle,\left\langle x_{1}, 0\right\rangle\right) \\
& =\left(\left\langle x_{2}, 0\right\rangle,\left\langle\bar{a} \bar{b} \bar{a} \bar{b}^{-1}, 0\right\rangle\right)=\tilde{a} \tilde{b} \tilde{a} \tilde{b}^{-1} .
\end{aligned}
$$

We set $B=\tilde{a} \tilde{b} \tilde{a} \tilde{b}^{-1}$. Then $\tilde{\alpha} \tilde{\beta} \tilde{\alpha} \tilde{\beta}^{-1}=B$. 
In order to compute $\tilde{\alpha} \tilde{a} \tilde{\alpha}^{-1}, \tilde{\alpha} \tilde{b} \tilde{\alpha}^{-1}, \tilde{\beta} \tilde{a} \tilde{\beta}^{-1}$ and $\tilde{\beta} \tilde{b} \tilde{\beta}^{-1}$ as elements of the kernel we consider the presentation $\pi_{1}(K, *)=\left\langle\varrho_{1}, \varrho_{2} \mid \varrho_{1}^{2} \varrho_{2}^{2}=1\right\rangle$.

Since the short exact sequence

$$
\begin{array}{r}
1 \rightarrow \pi_{1}\left(K \times K-\Delta_{K},\left(x_{2}, x_{1}\right)\right) \stackrel{i_{\#}}{\rightarrow} \pi_{1}\left(M(\phi) \times_{S^{1}} M(\phi)-\Delta,\left(\left\langle x_{2}, 0\right\rangle,\left\langle x_{1}, 0\right\rangle\right)\right) \\
\stackrel{p \circ p_{1 \mid \#}}{\longrightarrow} \pi_{1}\left(S^{1},\langle 0\rangle\right) \simeq \mathbb{Z} \rightarrow 1
\end{array}
$$

splits we have $\tilde{\alpha}=i_{\#}(\hat{\alpha}), \tilde{\beta}=i_{\#}(\hat{\beta}), \tilde{a}=i_{\#}(\hat{a})$ and $\tilde{b}=i_{\#}(\hat{b})$ where $\hat{\alpha}, \hat{\beta}, \hat{a}$ and $\hat{b}$ are the generators of $\pi_{1}\left(K \times K-\Delta_{K},\left(x_{2}, x_{1}\right)\right)$.

If we denote by $j_{\#}: \pi_{1}\left(K-x_{2}, x_{1}\right) \rightarrow \pi_{1}\left(K, x_{1}\right)$ the homomorphism induced by the inclusion $j: K-x_{2} \rightarrow K$, and by $k_{\#}: \pi_{1}\left(K-x_{1}, x_{2}\right) \rightarrow$ $\pi_{1}\left(K, x_{2}\right)$ the homomorphism induced by the inclusion $k: K-x_{1} \rightarrow K$ then $\bar{a}=\varrho_{11} \varrho_{12}, \bar{b}=\varrho_{12}^{-1}, \bar{\alpha}=\varrho_{21} \varrho_{22}$ and $\bar{\beta}=\varrho_{22}^{-1}$ where $\varrho_{11}, \varrho_{12}$ are the generators of $\pi_{1}\left(K-x_{2}, x_{1}\right)$ such that $j_{\#}\left(\varrho_{11}\right)=\varrho_{1}$ and $j_{\#}\left(\varrho_{12}\right)=\varrho_{2}$, and $\varrho_{21}, \varrho_{22}$ are the generators of $\pi_{1}\left(K-x_{1}, x_{2}\right)$ such that $k_{\#}\left(\varrho_{21}\right)=\varrho_{1}$ and $k_{\#}\left(\varrho_{22}\right)=\varrho_{2}$.

Also, if we denote by $\left(i_{2}\right)_{\#}: \pi_{1}\left(K-x_{2}, x_{1}\right) \rightarrow \pi_{1}\left(K \times K-\Delta_{K},\left(x_{2}, x_{1}\right)\right)$ the homomorphism induced by $i_{2}: K-x_{2} \rightarrow K \times K-\Delta_{K}$ given by $i_{2}(x)=\left(x_{2}, x\right)$, and by $\left(i_{1}\right)_{\#}$ the homomorphism induced by $i_{1}: K-x_{1} \rightarrow$ $K \times K-\Delta_{K}$ given by $i_{1}(x)=\left(x, x_{1}\right)$, then $\hat{a}=\left(i_{2}\right)_{\#}\left(\varrho_{11} \varrho_{12}\right), \hat{b}=\left(i_{2}\right)_{\#}\left(\varrho_{12}^{-1}\right)$, $\hat{\alpha}=\left(i_{1}\right)_{\#}\left(\varrho_{21} \varrho_{22}\right)$ and $\hat{\beta}=\left(i_{1}\right)_{\#}\left(\varrho_{22}^{-1}\right)$, or equivalently, $\hat{a}=\left(i_{2}\right)_{\#}(\bar{a})$, $\hat{b}=\left(i_{2}\right)_{\#}(\bar{b}), \hat{\alpha}=\left(i_{1}\right)_{\#}(\bar{\alpha})$ and $\hat{\beta}=\left(i_{1}\right)_{\#}(\bar{\beta})$ where $\bar{a}, \bar{b}, \bar{\alpha}$ and $\bar{\beta}$ are the generators of $\pi_{1}\left(K-x_{2}, x_{1}\right)$ and $\pi_{1}\left(K-x_{1}, x_{2}\right)$, respectively.

Let $B_{12}$ be as given in Figure 2 of Section 3, and $B_{21}=B_{12}^{-1}$.

Using [S69] and the convention that the product $c d$ of two elements in $\pi_{1}$ is the class of a representative of $c$ followed by a representative of $d$ we obtain the following presentation for $\pi_{1}\left(K \times K-\Delta_{K},\left(x_{2}, x_{1}\right)\right)$ :

$$
\begin{array}{ll}
B_{12}=\varrho_{11}^{2} \varrho_{12}^{2}, & B_{21}^{-1}=\varrho_{21}^{2} \varrho_{22}^{2}, \\
\varrho_{21} \varrho_{11} \varrho_{21}^{-1}=\varrho_{11} B_{12}^{-1}, & \varrho_{21} \varrho_{12} \varrho_{21}^{-1}=B_{12} \varrho_{11}^{-1} B_{12} \varrho_{11} \varrho_{12} \varrho_{11}^{-1} B_{12}^{-1} \varrho_{11} B_{12}^{-1}, \\
\varrho_{21} B_{12} \varrho_{21}^{-1}=B_{12} \varrho_{11}^{-1} B_{12}^{-1} \varrho_{11} B_{12}^{-1}, & \varrho_{22} \varrho_{11} \varrho_{22}^{-1}=\varrho_{11}, \\
\varrho_{22} \varrho_{12} \varrho_{22}^{-1}=\varrho_{12} B_{12}^{-1}, & \varrho_{22} B_{12} \varrho_{22}^{-1}=B_{12} \varrho_{12}^{-1} B_{12}^{-1} \varrho_{12} B_{12}^{-1}, \\
\varrho_{21}^{-1} \varrho_{11} \varrho_{21}=\varrho_{11}^{2} B_{12}^{-1} \varrho_{11}^{-1}, & \varrho_{21}^{-1} \varrho_{12} \varrho_{21}=\varrho_{11} B_{12} \varrho_{11}^{-1} B_{12} \varrho_{12} B_{12}^{-1} \varrho_{11} B_{12}^{-1} \varrho_{11}^{-1}, \\
\varrho_{21}^{-1} B_{12} \varrho_{21}=\varrho_{11} B_{12}^{-1} \varrho_{11}^{-1}, & \varrho_{22}^{-1} \varrho_{11} \varrho_{22}=\varrho_{11}, \\
\varrho_{22}^{-1} \varrho_{12} \varrho_{22}=\varrho_{12}^{2} B_{12}^{-1} \varrho_{12}^{-1}, & \varrho_{22}^{-1} B_{12} \varrho_{22}=\varrho_{12} B_{12}^{-1} \varrho_{12}^{-1} .
\end{array}
$$

We have $\hat{\alpha} \hat{a} \hat{\alpha}^{-1}=\left(i_{1}\right)_{\#}\left(\varrho_{21} \varrho_{22}\right)\left(i_{2}\right)_{\#}\left(\varrho_{11} \varrho_{12}\right)\left(i_{1}\right)_{\#}\left(\varrho_{22}^{-1} \varrho_{21}^{-1}\right)$. Using the relations above we obtain $\varrho_{21}\left(\varrho_{22}\left(\varrho_{11} \varrho_{12}\right) \varrho_{22}^{-1}\right) \varrho_{21}^{-1}=\varrho_{21}\left(\varrho_{11} \varrho_{12} B_{12}^{-1}\right) \varrho_{21}^{-1}=$ $B_{12} \varrho_{11} \varrho_{12} B_{12}^{-1}=\hat{B} \hat{a} \hat{B}^{-1}$. Therefore $\tilde{\alpha} \tilde{a} \tilde{\alpha}^{-1}=B \tilde{a} B^{-1}$.

Similarly we obtain all the other relations of the table (4.1). 
The fiber bundle

$$
\left(K \times K-\Delta_{K},\left(x_{2}, x_{1}\right)\right) \stackrel{i}{\rightarrow}\left(M(\phi) \times_{S^{1}} M(\phi)-\Delta,\left(\left\langle x_{2}, 0\right\rangle,\left\langle x_{1}, 0\right\rangle\right)\right) \stackrel{p \circ p_{1 \mid}}{\longrightarrow} S^{1},
$$

where $i(x, y)=(\langle x, 0\rangle,\langle y, 0\rangle)$, provides the short exact sequence

$$
\begin{aligned}
& 1 \rightarrow \pi_{1}\left(K \times K-\Delta_{K},\left(x_{2}, x_{1}\right)\right) \stackrel{i_{\#}}{\longrightarrow} \pi_{1}\left(M(\phi) \times_{S^{1}} M(\phi)-\Delta,\left(\left\langle x_{2}, 0\right\rangle,\left\langle x_{1}, 0\right\rangle\right)\right) \\
& \stackrel{p \circ p_{1 \mid \#}}{\longrightarrow} \pi_{1}\left(S^{1},\langle 0\rangle\right) \simeq \mathbb{Z} \rightarrow 1,
\end{aligned}
$$

which splits since the quotient is $\mathbb{Z}$. The action of $\mathbb{Z} \rightarrow \operatorname{Aut}\left(\pi_{1}(K \times K-\right.$ $\left.\left.\Delta_{K},\left(x_{2}, x_{1}\right)\right)\right)$ which comes from the section $s_{0}$ is given by $c \cdot \varrho=c \varrho c^{-1}=$ $(\phi \times \phi)_{\mid \#}(\varrho)$, where $c=p \circ p_{1 \mid \#}\left\langle s_{0}\right\rangle$ is the generator of $\pi_{1}\left(S^{1}\right)$, hence $\pi_{1}\left(M(\phi) \times_{S^{1}} M(\phi)-\Delta,\left(\left\langle x_{2}, 0\right\rangle,\left\langle x_{1}, 0\right\rangle\right)\right)$ is isomorphic to the semidirect product $\pi_{1}\left(K \times K-\Delta_{K},\left(x_{2}, x_{1}\right)\right) \rtimes \mathbb{Z}$.

Using the homeomorphism $h: M(\phi) \times{ }_{S^{1}} M(\phi) \rightarrow M(\phi \times \phi)$ over $S^{1}$ given by $h(\langle x, t\rangle,\langle y, t\rangle)=\langle x, y, t\rangle$ we obtain by restriction the homeomorphism $h_{\mid}: M(\phi) \times_{S^{1}} M(\phi)-\Delta \rightarrow M(\phi \times \phi)-h(\Delta)$ over $S^{1}$.

In $M(\phi \times \phi)-h(\Delta)$ the loops $\tilde{\alpha}, \tilde{\beta}, \tilde{c}_{0}, \tilde{a}, \tilde{b}$ can be seen as $\tilde{\alpha}=\left\langle\bar{\alpha}, x_{1}, 0\right\rangle$, $\tilde{\beta}=\left\langle\bar{\beta}, x_{1}, 0\right\rangle, \tilde{c}_{0}=\left\langle x_{2}, x_{1}, t\right\rangle, \tilde{a}=\left\langle x_{2}, \bar{a}, 0\right\rangle$ and $\tilde{b}=\left\langle x_{2}, \bar{b}, 0\right\rangle$, and they can be viewed as classes of the representative loops in $K \times K-\Delta_{K}$ of natural form.

So the loops $\tilde{\alpha}, \tilde{\beta}, \tilde{c}_{0}, \tilde{a}, \tilde{b}$ can be interpreted as follows: $\tilde{a}, \tilde{b}$ are elements in the second copy $K$ of $K \times K$ and $\tilde{\alpha}, \tilde{\beta}$ are elements in the first copy $K$ of $K \times K$. So

$$
\begin{aligned}
\tilde{c}_{0} \tilde{\alpha} \tilde{c}_{0}^{-1} \tilde{\alpha}^{-1} & =(\phi \times \phi)_{\mid \#}(\tilde{\alpha}) \tilde{\alpha}^{-1}=p_{2}(\tilde{a}, \tilde{b}), \\
\tilde{c}_{0} \tilde{\beta} \tilde{c}_{0}^{-1} \tilde{\beta}^{-\eta} \tilde{\alpha}^{-p} & =(\phi \times \phi)_{\mid \#}(\tilde{\beta}) \tilde{\beta}^{-\eta} \tilde{\alpha}^{-p}=p_{3}(\tilde{a}, \tilde{b}), \\
\tilde{c}_{0} \tilde{a} \tilde{c}_{0}^{-1} & =(\phi \times \phi)_{\mid \#}(\tilde{a}), \\
\tilde{c}_{0} \tilde{b} \tilde{c}_{0}^{-1} & =(\phi \times \phi)_{\mid \#}(\tilde{b}) .
\end{aligned}
$$

Now we are going to calculate

$$
(\phi \times \phi)_{\mid \#}: \pi_{1}\left(K \times K-\Delta_{K},\left(x_{2}, x_{1}\right)\right) \rightarrow \pi_{1}\left(K \times K-\Delta_{K},\left(x_{2}, x_{1}\right)\right) .
$$

For this:

(i) We consider the commutative diagrams

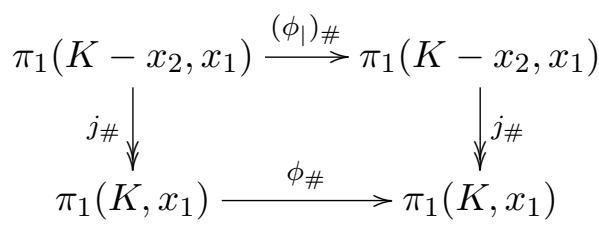


and

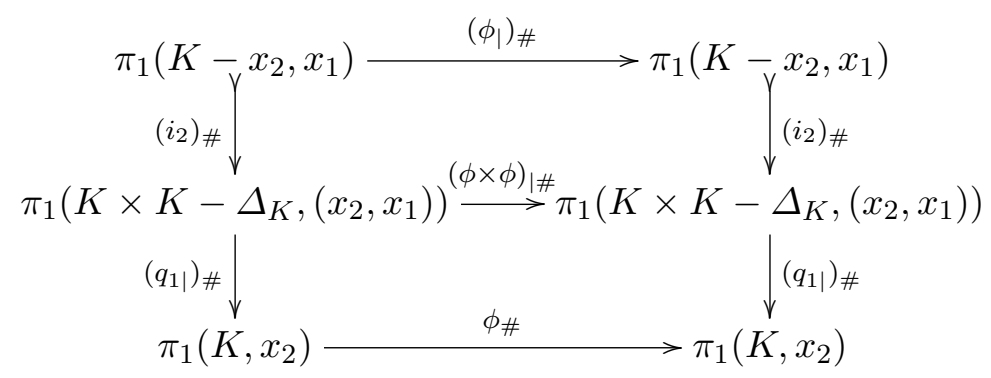

where $j_{\#}$ is induced by the inclusion $j: K-x_{2} \rightarrow K,\left(i_{2}\right)_{\#}$ is induced by $i_{2}: K-x_{2} \rightarrow K \times K-\Delta_{K}$ given by $i_{2}(x)=\left(x_{2}, x\right)$, and $\left(q_{1 \mid}\right)_{\#}$ is induced by $q_{1 \mid}: K \times K-\Delta_{K} \rightarrow K$ given by $q_{1 \mid}(x, y)=x$.

(ii) We denote by $\hat{a}, \hat{b}, \hat{\alpha}$ and $\hat{\beta}$ the generators of $\pi_{1}\left(K \times K-\Delta_{K},\left(x_{2}, x_{1}\right)\right)$ such that $i_{\#}(\hat{a})=\tilde{a}, i_{\#}(\hat{b})=\tilde{b}, i_{\#}(\hat{\alpha})=\tilde{\alpha}, i_{\#}(\hat{\beta})=\tilde{\beta}$ and $i_{2 \#}(\bar{a})$ $=\hat{a}, i_{2 \#}(\bar{b})=\hat{b}, i_{1 \#}(\bar{\alpha})=\hat{\alpha}, i_{1 \#}(\bar{\beta})=\hat{\beta}$.

(iii) We consider the identities

$$
\begin{aligned}
& \tilde{c}_{0} \tilde{\alpha}\left(\tilde{c}_{0}^{-1} \tilde{a} \tilde{c}_{0}\right) \tilde{\alpha}^{-1} \tilde{c}_{0}^{-1}=\left(\tilde{c}_{0} \tilde{\alpha} \tilde{c}_{0}^{-1}\right) \tilde{a}\left(\tilde{c}_{0} \tilde{\alpha}^{-1} \tilde{c}_{0}^{-1}\right), \\
& \tilde{c}_{0} \tilde{\alpha}\left(\tilde{c}_{0}^{-1} \tilde{b} \tilde{c}_{0}\right) \tilde{\alpha}^{-1} \tilde{c}_{0}^{-1}=\left(\tilde{c}_{0} \tilde{\alpha} \tilde{c}_{0}^{-1}\right) \tilde{b}\left(\tilde{c}_{0} \tilde{\alpha}^{-1} \tilde{c}_{0}^{-1}\right), \\
& \tilde{c}_{0} \tilde{\beta}\left(\tilde{c}_{0}^{-1} \tilde{a} \tilde{c}_{0}\right) \tilde{\beta}^{-1} \tilde{c}_{0}^{-1}=\left(\tilde{c}_{0} \tilde{\beta} \tilde{c}_{0}^{-1}\right) \tilde{a}\left(\tilde{c}_{0} \tilde{\beta}^{-1} \tilde{c}_{0}^{-1}\right), \\
& \tilde{c}_{0} \tilde{\beta}\left(\tilde{c}_{0}^{-1} \tilde{b}_{0}\right) \tilde{\beta}^{-1} \tilde{c}_{0}^{-1}=\left(\tilde{c}_{0} \tilde{\beta} \tilde{c}_{0}^{-1}\right) \tilde{b}\left(\tilde{c}_{0} \tilde{\beta}^{-1} \tilde{c}_{0}^{-1}\right) .
\end{aligned}
$$

(iv) We write $\pi_{1}(K, *)=\left\langle\bar{a}, \bar{b} \mid \bar{a} \bar{b} \bar{a} \bar{b}^{-1}=1\right\rangle$ and $\pi_{1}(K-x, *)=\langle a, b\rangle$. If $w_{a}, w_{b} \in \pi_{1}(K-x, *)$ are such that $w_{a} w_{b} w_{a} w_{b}^{-1}=a b a b^{-1}$ or $w_{a} w_{b} w_{a} w_{b}^{-1}=b a^{-1} b^{-1} a^{-1}$ then by Theorem 1.1 of [Sko87] there exists a homeomorphism $\phi: K \rightarrow K$ such that $\left(\phi_{\mid}\right)_{\#}$ maps $a \mapsto w_{a}$ and $b \mapsto w_{b}$.

Now we are going to use the above diagrams in each of the four cases.

CASE I: $\phi_{0}(1,1)$. In this case let $\phi: K \rightarrow K$ be the identity. We have $\left(\phi_{\mid}\right)_{\#}: \bar{a} \mapsto \bar{a}, \bar{b} \mapsto \bar{b}$ and so $(\phi \times \phi)_{\mid \#}: \hat{a} \mapsto \hat{a}, \hat{b} \mapsto \hat{b}$. Therefore $\tilde{c}_{0} \tilde{a} \tilde{c}_{0}^{-1}=(\phi \times \phi)_{\mid \#}(\tilde{a})=(\phi)_{\mid \#}(\tilde{a})=\tilde{a}$ and $\tilde{c}_{0} \tilde{b} \tilde{c}_{0}^{-1}=(\phi \times \phi)_{\mid \#}(\tilde{b})=\tilde{b}$. Since in this case $\tilde{c}_{0}^{-1} \tilde{b} \tilde{c}_{0}=\tilde{b}$ and $\tilde{c}_{0}^{-1} \tilde{a} \tilde{c}_{0}=\tilde{a}$ it follows from the identities $\tilde{c}_{0} \tilde{\alpha}\left(\tilde{c}_{0}^{-1} \tilde{a} \tilde{c}_{0}\right) \tilde{\alpha}^{-1} \tilde{c}_{0}^{-1}=\left(\tilde{c}_{0} \tilde{\alpha} \tilde{c}_{0}^{-1}\right) \tilde{a}\left(\tilde{c}_{0} \tilde{\alpha}^{-1} \tilde{c}_{0}^{-1}\right)$ and $\tilde{c}_{0} \tilde{\alpha}\left(\tilde{c}_{0}^{-1} \tilde{b}_{0} \tilde{c}_{0}\right) \tilde{\alpha}^{-1} \tilde{c}_{0}^{-1}=$ $\left(\tilde{c}_{0} \tilde{\alpha} \tilde{c}_{0}^{-1}\right) \tilde{b}\left(\tilde{c}_{0} \tilde{\alpha}^{-1} \tilde{c}_{0}^{-1}\right)$ that $p_{2}(\tilde{a}, \tilde{b})$ must satisfy $B \tilde{a} B^{-1}=p_{2}(\tilde{a}, \tilde{b}) B \tilde{a} B^{-1}$ $p_{2}(\tilde{a}, \tilde{b})^{-1}$ and $B \tilde{a}^{-1}\left(\tilde{b} \tilde{a}^{-2}\right) \tilde{a} B^{-1}=p_{2}(\tilde{a}, \tilde{b}) B \tilde{a}^{-1}\left(\tilde{b} \tilde{a}^{-2}\right) \tilde{a} B^{-1} p_{2}(\tilde{a}, \tilde{b})^{-1}$. So $p_{2}(\tilde{a}, \tilde{b})$ commutes with the subgroup generated by $B \tilde{a} B^{-1}$ and $B \tilde{a}^{-1}\left(\tilde{b} \tilde{a}^{-2}\right)$ - $\tilde{a} B^{-1}$. Suppose that $p_{2}(\tilde{a}, \tilde{b}) \neq 1$. From Exercise 7 in Section 1.4 of [MKS66] we know that some nontrivial power of $B \tilde{a} B^{-1}$ is also a power of $p_{2}(\tilde{a}, \tilde{b})$. Similarly for $B \tilde{a}^{-1}\left(\tilde{b} \tilde{a}^{-2}\right) \tilde{a} B^{-1}$. By Exercise 4 in Section 1.4 of [MKS66] 
the two elements $B \tilde{a} B^{-1}$ and $B \tilde{a}^{-1}\left(\tilde{b} \tilde{a}^{-2}\right) \tilde{a} B^{-1}$ commute, so they generate a cyclic subgroup. But the subgroup generated by $B \tilde{a} B^{-1}$ and $B \tilde{a}^{-1}$ - $\left(\tilde{b} \tilde{a}^{-2}\right) \tilde{a} B^{-1}$ cannot be cyclic since it is not cyclic in the abelianization. So we get $p_{2}(\tilde{a}, \tilde{b})=1$. Also, from the identities $\tilde{c}_{0} \tilde{\beta}\left(\tilde{c}_{0}^{-1} \tilde{a} \tilde{c}_{0}\right) \tilde{\beta}^{-1} \tilde{c}_{0}^{-1}=$ $\left(\tilde{c}_{0} \tilde{\beta} \tilde{c}_{0}^{-1}\right) \tilde{a}\left(\tilde{c}_{0} \tilde{\beta}^{-1} \tilde{c}_{0}^{-1}\right)$ and $\tilde{c}_{0} \tilde{\beta}\left(\tilde{c}_{0}^{-1} \tilde{b} \tilde{c}_{0}\right) \tilde{\beta}^{-1} \tilde{c}_{0}^{-1}=\left(\tilde{c}_{0} \tilde{\beta} \tilde{c}_{0}^{-1}\right) \tilde{b}\left(\tilde{c}_{0} \tilde{\beta}^{-1} \tilde{c}_{0}^{-1}\right)$ we find that $p_{3}(\tilde{a}, \tilde{b})$ must satisfy $\tilde{b}^{-1} \tilde{a}^{-1} \tilde{b}=p_{3}(\tilde{a}, \tilde{b}) \tilde{b}^{-1} \tilde{a}^{-1} \tilde{b}+p_{3}(\tilde{a}, \tilde{b})^{-1}$ and $\tilde{b}^{-1}(B \tilde{b}) \tilde{b}$ $=p_{3}(\tilde{a}, \tilde{b}) \tilde{b}^{-1}(B \tilde{b}) \tilde{b} p_{3}(\tilde{a}, \tilde{b})^{-1}$. So $p_{3}(\tilde{a}, \tilde{b})=1$ is the unique solution. Therefore $\tilde{c}_{0} \tilde{\alpha} \tilde{c}_{0}^{-1} \tilde{\alpha}^{-1}=p_{2}(\tilde{a}, \tilde{b})=1$ and $\tilde{c}_{0} \tilde{\beta} \tilde{c}_{0}^{-1} \tilde{\beta}^{-1}=p_{3}(\tilde{a}, \tilde{b})=1$. As $\tilde{c}_{0} \tilde{\alpha} \tilde{c}_{0}^{-1} \tilde{\alpha}^{-1}$ $=(\phi \times \phi)_{\mid \#}(\tilde{\alpha}) \tilde{\alpha}^{-1}=1$ and $\tilde{c}_{0} \tilde{\beta} \tilde{c}_{0}^{-1} \tilde{\beta}^{-1}=(\phi \times \phi)_{\mid \#}(\tilde{\beta}) \tilde{\beta}^{-1}=1$ it follows that $(\phi \times \phi)_{\mid \#}(\tilde{\alpha})=\tilde{\alpha}$ and $(\phi \times \phi)_{\mid \#}(\tilde{\beta})=\tilde{\beta}$.

CAse II: $\phi_{1}(1,1)$. By (iv) we can take $\phi$ such that $\left(\phi_{\mid}\right)_{\#}: \bar{a} \mapsto \bar{a}, \bar{b} \mapsto$ $\bar{b} \bar{a}^{-1}$. Now, by diagrams in (i) we have $\phi_{\#}=\phi_{1}(1,1)$ and $(\phi \times \phi)_{\mid \#}: \hat{a} \mapsto \hat{a}$, $\hat{b} \mapsto \hat{b} \hat{a}^{-1}$. So $\tilde{c}_{0} \tilde{a} \tilde{c}_{0}^{-1}=(\phi \times \phi)_{\mid \#}(\tilde{a})=\tilde{a}$ and $\tilde{c}_{0} \tilde{b} \tilde{c}_{0}^{-1}=(\phi \times \phi)_{\mid \#}(\tilde{b})=\tilde{b} \tilde{a}^{-1}$. Now, as in Case I, it follows from the identities (iii) that $p_{2}(\tilde{a}, \tilde{b})=1$ and $p_{3}(\tilde{a}, \tilde{b})=B^{-1}$ are the unique solutions. Therefore $\tilde{c}_{0} \tilde{\alpha} \tilde{c}_{0}^{-1} \tilde{\alpha}^{-1}=p_{2}(\tilde{a}, \tilde{b})=1$ and $\tilde{c}_{0} \tilde{\beta} \tilde{c}_{0}^{-1} \tilde{\beta}^{-1} \tilde{\alpha}^{-1}=p_{3}(\tilde{a}, \tilde{b})=B^{-1}$. As $\tilde{c}_{0} \tilde{\alpha} \tilde{c}_{0}^{-1} \tilde{\alpha}^{-1}=(\phi \times \phi)_{\mid \#}(\tilde{\alpha}) \tilde{\alpha}^{-1}=1$ and $\tilde{c}_{0} \tilde{\beta} \tilde{c}_{0}^{-1} \tilde{\beta}^{-1} \tilde{\alpha}^{-1}=(\phi \times \phi)_{\mid \#}(\tilde{\beta}) \tilde{\beta}^{-1} \tilde{\alpha}^{-1}=B^{-1}$ where $B=\tilde{a} \tilde{b} \tilde{a} \tilde{b}^{-1}$ it follows that $(\phi \times \phi)_{\mid \#}(\tilde{\alpha})=\tilde{\alpha}$ and $(\phi \times \phi)_{\mid \#}(\tilde{\beta})=B^{-1} \tilde{\alpha} \tilde{\beta}$.

CASE III: $\phi_{0}(1,-1)$. By (iv) we can take $\phi$ such that $\left(\phi_{\mid}\right)_{\#}: \bar{a} \mapsto$ $\bar{a} \bar{b} \bar{a}^{-1} \bar{b}^{-1} \bar{a}^{-1}=\bar{a} \bar{E}^{-1}, \bar{b} \mapsto \bar{a} \bar{b} \bar{a} \bar{b}^{-1} \bar{a}^{-1} \bar{b}^{-1} \bar{a}^{-1}=\bar{E} \bar{b}^{-1} \bar{E}^{-1}$ where $\bar{E}=$ $\bar{a} \bar{b} \bar{a} \bar{b}^{-1}$. Now, by diagrams in (i) we have $\phi_{\#}=\phi_{0}(1,-1)$ and $(\phi \times \phi)_{\mid \#}$ : $\hat{a} \mapsto \hat{a} \hat{b} \hat{a}^{-1} \hat{b}^{-1} \hat{a}^{-1}=\hat{a} \hat{E}^{-1}, \hat{b} \mapsto \hat{a} \hat{b} \hat{a} \hat{b}^{-1} \hat{a}^{-1} \hat{b}^{-1} \hat{a}^{-1}=\hat{E} \hat{b}^{-1} \hat{E}^{-1}$ where $\hat{E}=\hat{a} \hat{b} \hat{a} \hat{b}^{-1}$. Therefore $\tilde{c}_{0} \tilde{a} \tilde{c}_{0}^{-1}=(\phi \times \phi)_{\mid \#}(\tilde{a})=\tilde{a} \tilde{b} \tilde{a}^{-1} \tilde{b}^{-1} \tilde{a}^{-1}=\tilde{a} B^{-1}$ and $\tilde{c}_{0} \tilde{b} \tilde{c}_{0}^{-1}=(\phi \times \phi)_{\mid \#}(\tilde{b})=\tilde{a} \tilde{b} \tilde{a} \tilde{b}^{-1} \tilde{a}^{-1} \tilde{b}^{-1} \tilde{a}^{-1}=B \tilde{b}^{-1} B^{-1}$ where $B=\tilde{a} \tilde{b} \tilde{a} \tilde{b}^{-1}$. Now, as in Case I, it follows from the identities (iii) that $p_{2}(\tilde{a}, \tilde{b})=B^{-1}$ and $p_{3}(\tilde{a}, \tilde{b})=1$ are the unique solutions. Therefore $\tilde{c}_{0} \tilde{\alpha} \tilde{c}_{0}^{-1} \tilde{\alpha}^{-1}=p_{2}(\tilde{a}, \tilde{b})=B^{-1}$ and $\tilde{c}_{0} \tilde{\beta} \tilde{c}_{0}^{-1} \tilde{\beta}=p_{3}(\tilde{a}, \tilde{b})=1$. As $\tilde{c}_{0} \tilde{\alpha} \tilde{c}_{0}^{-1} \tilde{\alpha}^{-1}=(\phi \times \phi)_{\mid \#}(\tilde{\alpha}) \tilde{\alpha}^{-1}=B^{-1}$ and $\tilde{c}_{0} \tilde{\beta} \tilde{c}_{0}^{-1} \tilde{\beta}=(\phi \times \phi)_{\mid \#}(\tilde{\beta}) \tilde{\beta}=1$ it follows that $(\phi \times \phi)_{\mid \#}(\tilde{\alpha})=B^{-1} \tilde{\alpha}$ and $(\phi \times \phi)_{\mid \#}(\tilde{\beta})=\tilde{\beta}^{-1}$.

CASE IV: $\phi_{1}(1,-1)$. By (iv) we can take $\phi$ such that $\left(\phi_{\mid}\right)_{\#}: \bar{a} \mapsto \bar{a} \bar{E}^{-1}$, $\bar{b} \mapsto \bar{E} \bar{b}^{-1} \bar{a}^{-1}$ where $\bar{E}=\bar{a} \bar{b} \bar{a} \bar{b}^{-1}$. Now, by diagrams in (i) we have $\phi_{\#}=$ $\phi_{1}(1,-1)$ and $(\phi \times \phi)_{\mid \#}: \hat{a} \mapsto \hat{a} \hat{E}^{-1}, \hat{b} \mapsto \hat{E} \hat{b}^{-1} \hat{a}^{-1}$ where $\hat{E}=\hat{a} \hat{b} \hat{a} \hat{b}^{-1}$. Therefore $\tilde{c}_{0} \tilde{a} \tilde{c}_{0}^{-1}=(\phi \times \phi)_{\mid \#}(\tilde{a})=\tilde{a} B^{-1}$ and $\tilde{c}_{0} \tilde{b} \tilde{c}_{0}^{-1}=(\phi \times \phi)_{\mid \#}(\tilde{b})=B \tilde{b}^{-1} \tilde{a}^{-1}$. So, it follows from the identities (iii) that $p_{2}(\tilde{a}, \tilde{b})=B^{-1}$ and $p_{3}(\tilde{a}, \tilde{b})$ $=1$ are the unique solutions. Therefore $\tilde{c}_{0} \tilde{\alpha} \tilde{c}_{0}^{-1} \tilde{\alpha}^{-1}=p_{2}(\tilde{a}, \tilde{b})=B^{-1}$ and $\tilde{c}_{0} \tilde{\beta} \tilde{c}_{0}^{-1} \tilde{\beta} \tilde{\alpha}^{-1}=p_{3}(\tilde{a}, \tilde{b})=1$. As $\tilde{c}_{0} \tilde{\alpha} \tilde{c}_{0}^{-1} \tilde{\alpha}^{-1}=(\phi \times \phi)_{\mid \#}(\tilde{\alpha}) \tilde{\alpha}^{-1}=B^{-1}$ and 
$\tilde{c}_{0} \tilde{\beta} \tilde{c}_{0}^{-1} \tilde{\beta} \tilde{\alpha}^{-1}=(\phi \times \phi)_{\mid \#}(\tilde{\beta}) \tilde{\beta} \tilde{\alpha}^{-1}=1$ it follows that $(\phi \times \phi)_{\mid \#}(\tilde{\alpha})=B^{-1} \tilde{\alpha}$ and $(\phi \times \phi)_{\mid \#}(\tilde{\beta})=\tilde{\alpha} \tilde{\beta}^{-1}$. This settlesall the cases from the table (4.2).

REMARK 4.1. We observe that every word $p(\tilde{a}, \tilde{b})$ can be written as a word in $w=\tilde{a} \tilde{b} \tilde{a}^{-1} \tilde{b}^{-1} \tilde{a}^{-1}$ and $v=\tilde{a} \tilde{b}$ because $\tilde{a}=v^{-1} w^{-1} v$ and $\tilde{b}=v^{-1} w v^{2}$. For the next sections it will be convenient to calculate the conjugates of the generators $v$ and $w$ by $\tilde{\alpha}, \tilde{\beta}$ and $\tilde{c}_{0}$. They are given in the tables (4.3) and (4.4) below.

\begin{tabular}{|l|l|}
\hline$\tilde{\alpha} v \tilde{\alpha}^{-1}=w v w$ & $\tilde{\alpha}^{-1} v \tilde{\alpha}=w^{-1} v w^{-1}$ \\
\hline$\tilde{\alpha} w \tilde{\alpha}^{-1}=w$ & $\tilde{\alpha}^{-1} w \tilde{\alpha}=w$ \\
\hline$\tilde{\alpha} B \tilde{\alpha}^{-1}=\tilde{\alpha}\left(w^{-1} v^{-1} w^{-1} v\right) \tilde{\alpha}^{-1}$ & \begin{tabular}{rl|}
$\tilde{\alpha}^{-1} B \tilde{\alpha}=\tilde{\alpha}^{-1}\left(w^{-1} v^{-1} w^{-1} v\right) \tilde{\alpha}$ \\
\\
$\quad=w^{-1} B w$ & \\
& $=w B w^{-1}$
\end{tabular} \\
\hline$\tilde{\beta} v \tilde{\beta}^{-1}=v$ & $\tilde{\beta}^{-1} v \tilde{\beta}=v$ \\
\hline$\tilde{\beta} w \tilde{\beta}^{-1}=v^{-1} w^{-1} v$ & $\tilde{\beta}^{-1} w \tilde{\beta}=v w^{-1} v^{-1}$ \\
\hline$\tilde{\beta} B \tilde{\beta}^{-1}=v^{-1} w B^{-1} w^{-1} v$ & $\tilde{\beta}^{-1} B \tilde{\beta}=v w B^{-1} w^{-1} v^{-1}$ \\
\hline
\end{tabular}

\begin{tabular}{|c|rcc|}
\hline Case I & $\tilde{c}_{0} v \tilde{c}_{0}^{-1}=v$ & $\tilde{c}_{0} w \tilde{c}_{0}^{-1}=w$ & $\tilde{c}_{0} B \tilde{c}_{0}^{-1}=B$ \\
$\phi_{0}(1,1)$ & $\tilde{c}_{0}^{-1} v \tilde{c}_{0}=v$ & $\tilde{c}_{0}^{-1} w \tilde{c}_{0}=w$ & $\tilde{c}_{0}^{-1} B \tilde{c}_{0}=B$ \\
\hline Case II & $\tilde{c}_{0} v \tilde{c}_{0}^{-1}=w v$ & $\tilde{c}_{0} w \tilde{c}_{0}^{-1}=w$ & $\tilde{c}_{0} B \tilde{c}_{0}^{-1}=B$ \\
$\phi_{1}(1,1)$ & $\tilde{c}_{0}^{-1} v \tilde{c}_{0}=w^{-1} v$ & $\tilde{c}_{0}^{-1} w \tilde{c}_{0}=w$ & $\tilde{c}_{0}^{-1} B \tilde{c}_{0}=B$ \\
\hline Case III & $\tilde{c}_{0} v \tilde{c}_{0}^{-1}=v^{-1}$ & $\tilde{c}_{0} w \tilde{c}_{0}^{-1}=v^{-1} w^{-1} v$ & $\tilde{c}_{0} B \tilde{c}_{0}^{-1}=B^{-1}$ \\
$\phi_{0}(1,-1)$ & $\tilde{c}_{0}^{-1} v \tilde{c}_{0}=v^{-1}$ & $\tilde{c}_{0}^{-1} w \tilde{c}_{0}=v^{-1} w^{-1} v$ & $\tilde{c}_{0}^{-1} B \tilde{c}_{0}=B^{-1}$ \\
\hline Case IV & $\tilde{c}_{0} v \tilde{c}_{0}^{-1}=v^{-1} w^{-1}$ & $\tilde{c}_{0} w \tilde{c}_{0}^{-1}=v^{-1} w^{-1} v$ & $\tilde{c}_{0} B \tilde{c}_{0}^{-1}=B^{-1}$ \\
$\phi_{1}(1,-1)$ & $\tilde{c}_{0}^{-1} v \tilde{c}_{0}=v^{-1} w$ & $\tilde{c}_{0}^{-1} w \tilde{c}_{0}=v^{-1} w^{-1} v$ & $\tilde{c}_{0}^{-1} B \tilde{c}_{0}=B^{-1}$ \\
\hline
\end{tabular}

5. The lifting problem and some reductions. Now we study the existence of the lift in the algebraic diagram (1.1) given in Subsection 1.1.

The homomorphism $j_{2 \#}$ is the induced by the map

$$
j_{2}:\left(K-x_{2}, x_{1}\right) \rightarrow\left(M(\phi) \times_{S^{1}} M(\phi)-\Delta,\left(\left\langle x_{2}, 0\right\rangle,\left\langle x_{1}, 0\right\rangle\right)\right)
$$

given by $y \mapsto\left(\left\langle x_{2}, 0\right\rangle,\langle y, 0\rangle\right)$. The homomorphism $\partial_{2}$ is the connecting homomorphism of the homotopy exact sequence of the pair $\left(K, K-x_{2}\right)$,

$$
1 \rightarrow \pi_{2}\left(K, K-x_{2}, x_{1}\right) \stackrel{\partial_{2}}{\longrightarrow} \pi_{1}\left(K-x_{2}, x_{1}\right) \stackrel{j_{\pi}}{\longrightarrow} \pi_{1}\left(K, x_{1}\right) \rightarrow 1
$$

and the homomorphism

$$
\begin{gathered}
\pi_{1}\left(M(\phi) \times_{S^{1}} M(\phi)-\Delta,\left(\left\langle x_{2}, 0\right\rangle,\left\langle x_{1}, 0\right\rangle\right)\right) \\
\downarrow^{q_{\#}} \\
\pi_{1}\left(M(\phi) \times_{S^{1}} M(\phi),\left(\left\langle x_{2}, 0\right\rangle,\left\langle x_{2}, 0\right\rangle\right)\right)
\end{gathered}
$$


is given by the composition

$$
\begin{gathered}
\pi_{1}\left(M(\phi) \times_{S^{1}} M(\phi)-\Delta,\left(\left\langle x_{2}, 0\right\rangle,\left\langle x_{1}, 0\right\rangle\right)\right) \\
\kappa \downarrow \\
\pi_{1}\left(M(\phi) \times_{S^{1}} M(\phi),\left(\left\langle x_{2}, 0\right\rangle,\left\langle x_{1}, 0\right\rangle\right)\right) \\
\bar{\nu} \downarrow \simeq \\
\pi_{1}\left(M(\phi) \times_{S^{1}} M(\phi),\left(\left\langle x_{2}, 0\right\rangle,\left\langle x_{2}, 0\right\rangle\right)\right) .
\end{gathered}
$$

Here $\bar{\nu}(\eta)=\nu^{-1} \eta \nu$ where $\nu: I \rightarrow M(\phi) \times_{S^{1}} M(\phi)$ is given by $\nu(t)=$ $\left(\left\langle x_{2}, 0\right\rangle,\langle\sigma(t), 0\rangle\right)$ with $\sigma: I \rightarrow K$ a path joining $x_{1}$ to $x_{2}$ and $\kappa$ is the homomorphism given by $\tilde{\alpha} \mapsto \alpha_{1}, \tilde{\beta} \mapsto \beta_{1}, \tilde{c}_{0} \mapsto c_{01}, \tilde{a} \mapsto u_{1}, \tilde{b} \mapsto v_{1}$. In order to compute $\bar{\nu}^{-1} \circ(1, f)_{\#}$ we will compute the homomorphism $(1, f)_{\#}$ and the homomorphism $\bar{\nu}^{-1}$ of the above diagram, where $f_{\#}=$ $f_{s}\left(r, 1, c_{1}, c_{2}\right)$ is given in the table from Theorem 2.4. From Sections 1, 3 and 4 we have

$$
\begin{aligned}
& \pi_{1}\left(M(\phi),\left\langle x_{2}, 0\right\rangle\right)=\left\langle\alpha, \beta, c_{0} \mid \alpha \beta \alpha \beta^{-1}=1, c_{0} \alpha c_{0}^{-1}=\alpha, c_{0} \beta c_{0}^{-1}=\alpha^{p} \beta^{\eta}\right\rangle, \\
& \pi_{1}\left(M(\phi) \times_{S^{1}} M(\phi),\left(\left\langle x_{2}, 0\right\rangle,\left\langle x_{2}, 0\right\rangle\right)\right)=\left\langle\alpha_{2}, \beta_{2}, c_{02}, u_{2}, v_{2}\right| u_{2} v_{2} u_{2} v_{2}^{-1}=1, \\
& \alpha_{2} \beta_{2} \alpha_{2} \beta_{2}^{-1}=1, c_{02} \alpha_{2} c_{02}{ }^{-1} \alpha_{2}^{-1}=1, c_{02} \beta_{2} c_{02}^{-1} \beta_{2}^{-\eta} \alpha_{2}^{-p}=1, \alpha_{2} u_{2} \alpha_{2}^{-1}=u_{2}, \\
& \left.\alpha_{2} v_{2} \alpha_{2}^{-1}=v_{2}, \beta_{2} u_{2} \beta_{2}^{-1}=u_{2}, \beta_{2} v_{2} \beta_{2}^{-1}=v_{2}, c_{02} u_{2} c_{02}^{-1}=u_{2}^{\epsilon}, c_{02} v_{2} c_{02}^{-1}=u_{2}^{p} v_{2}{ }^{\eta}\right\rangle \\
& \pi_{1}\left(M(\phi) \times_{S^{1}} M(\phi)-\Delta,\left(\left\langle x_{2}, 0\right\rangle,\left\langle x_{1}, 0\right\rangle\right)\right) \\
& =\left\langle\tilde{\alpha}, \tilde{\beta}, \tilde{c}_{0}, \tilde{a}, \tilde{b}\right| \tilde{\alpha} \tilde{\beta} \tilde{\alpha} \tilde{\beta}^{-1}=\tilde{a} \tilde{b} \tilde{a} \tilde{b}^{-1}=B, \tilde{c}_{0} \tilde{\alpha} \tilde{c}_{0}^{-1} \tilde{\alpha}^{-\epsilon}=(\phi \times \phi)_{\mid \#}(\tilde{\alpha}) \tilde{\alpha}^{-\epsilon}, \\
& \tilde{c}_{0} \tilde{\beta} \tilde{c}_{0}^{-1} \tilde{\beta}^{-\eta} \tilde{\alpha}^{-p}=(\phi \times \phi)_{\mid \#}(\tilde{\beta}) \tilde{\beta}^{-\eta} \tilde{\alpha}^{-p}, \tilde{\alpha} \tilde{a} \tilde{\alpha}^{-1}=B \tilde{a} B^{-1}, \\
& \tilde{\alpha} \tilde{b} \tilde{\alpha}^{-1}=B \tilde{a} \tilde{a}^{-1} \tilde{b} \tilde{a}^{-1} B^{-1}, \tilde{\beta} \tilde{a} \tilde{\beta}^{-1}=\tilde{b}^{-1} \tilde{a}^{-1} \tilde{b}, \tilde{\beta} \tilde{b} \tilde{\beta}^{-1}=\tilde{b}^{-1}(B \tilde{b}) \tilde{b}, \\
& \left.\tilde{c}_{0} \tilde{a} \tilde{c}_{0}^{-1}=(\phi \times \phi)_{\mid \#}(\tilde{a}), \tilde{c}_{0} \tilde{b} \tilde{c}_{0}^{-1}=(\phi \times \phi)_{\mid \#}(\tilde{b})\right\rangle
\end{aligned}
$$

and

$\pi_{1}\left(M(\phi) \times_{S^{1}} M(\phi),\left(\left\langle x_{2}, 0\right\rangle,\left\langle x_{1}, 0\right\rangle\right)\right)=\left\langle\alpha_{1}, \beta_{1}, c_{01}, u_{1}, v_{1}\right| u_{1} v_{1} u_{1} v_{1}^{-1}=1$, $\alpha_{1} \beta_{1} \alpha_{1} \beta_{1}^{-1}=1, c_{01} \alpha_{1} c_{01}^{-1} \alpha_{1}^{-\epsilon}=1, c_{01} \beta_{1} c_{01}^{-1} \beta_{1}^{-\eta} \alpha_{1}^{-p}=1, \alpha_{1} u_{1} \alpha_{1}^{-1}=u_{1}$, $\left.\alpha_{1} v_{1} \alpha_{1}^{-1}=v_{1}, \beta_{1} u_{1} \beta_{1}^{-1}=u_{1}, \beta_{1} v_{1} \beta_{1}^{-1}=v_{1}, c_{01} u_{1} c_{01}^{-1}=u_{1}^{\epsilon}, c_{01} v_{1} c_{01}^{-1}=u_{1}^{p} v_{1}^{\eta}\right\rangle$, where $p \in\{0,1\}, \epsilon= \pm 1$ and $\eta= \pm 1$.

We have $(1, f)_{\#}(\langle\alpha(t)\rangle)=\langle(1, f) \circ \alpha(t)\rangle=\langle(\alpha(t), f \circ \alpha(t))\rangle$. But $(\alpha(t)$, $f \circ \alpha(t)) \sim_{\partial I}\left(\alpha(t),\left\langle x_{2}, 0\right\rangle\right) *\left(\left\langle x_{2}, 0\right\rangle, f \circ \alpha(t)\right)$. 
Since $f_{\#}(\alpha)=\alpha^{r}$ it follows that

$$
\begin{aligned}
(1, f)_{\#}(\langle\alpha\rangle) & =\langle(1, f) \circ \alpha\rangle=\langle(\alpha, f \circ \alpha)\rangle \\
& =\left\langle\left(\alpha,\left\langle x_{2}, 0\right\rangle\right)\right\rangle\left\langle\left(\left\langle x_{2}, 0\right\rangle, f \circ \alpha\right)\right\rangle \\
& =\left\langle\left(\alpha,\left\langle x_{2}, 0\right\rangle\right)\right\rangle\left\langle\left(\left\langle x_{2}, 0\right\rangle, \alpha^{r}\right)\right\rangle=\alpha_{2} u_{2}^{r} .
\end{aligned}
$$

Similarly, as $f_{\#}(\beta)=\alpha^{s} \beta$ we have $(1, f)_{\#}(\langle\beta\rangle)=\beta_{2} u_{2}^{s} v_{2}$. Also, $(1, f)_{\#}\left(\left\langle c_{0}\right\rangle\right)$ $=\left\langle(1, f) \circ c_{0}\right\rangle=\left\langle\left(c_{0}, f \circ c_{0}\right)\right\rangle$. But, since $f_{\#}\left(c_{0}\right)=\alpha^{c_{1}} \beta^{c_{2}} c_{0}$ we have $\left(c_{0}, f \circ c_{0}\right)$ $\simeq_{\partial I}\left(\left\langle x_{2}, 0\right\rangle, \alpha^{c_{1}}\right) *\left(\left\langle x_{2}, 0\right\rangle, \beta^{c_{2}}\right) *\left(c_{0}, c_{0}\right)$. So $(1, f)_{\#}\left(c_{0}\right)=u_{2}^{c_{1}} v_{2}^{c_{2}} c_{02}$. Summarizing, in all cases, if $f_{\#}=f_{s}\left(r, 1, c_{1}, c_{2}\right)$ then $(1, f)_{\#}$ is given by $\alpha \mapsto \alpha_{2} u_{2}^{r}$, $\beta \mapsto \beta_{2} u_{2}^{s} v_{2}$ and $c_{0} \mapsto u_{2}^{c_{1}} v_{2}^{c_{2}} c_{02}$.

For

$$
\bar{\nu}: \pi_{1}\left(M(\phi) \times_{S^{1}} M(\phi),\left(\left\langle x_{2}, 0\right\rangle,\left\langle x_{1}, 0\right\rangle\right)\right) \rightarrow \pi_{1}\left(M(\phi) \times_{S^{1}} M(\phi),\left(\left\langle x_{2}, 0\right\rangle,\left\langle x_{2}, 0\right\rangle\right)\right)
$$

we have

$$
\begin{aligned}
\bar{\nu}\left(\alpha_{1}\right) & =\nu^{-1} \alpha_{1} \nu=\left(\left\langle x_{2}, 0\right\rangle,\left\langle\sigma^{-1}, 0\right\rangle\right)\left(\alpha,\left\langle x_{1}, 0\right\rangle\right)\left(\left\langle x_{2}, 0\right\rangle,\langle\sigma, 0\rangle\right) \\
& =\left(\alpha,\left\langle x_{2}, 0\right\rangle\right)=\alpha_{2} .
\end{aligned}
$$

Similarly $\bar{\nu}\left(\beta_{1}\right)=\beta_{2}, \bar{\nu}\left(c_{01}\right)=c_{02}, \bar{\nu}\left(u_{1}\right)=u_{2}$ and $\bar{\nu}\left(v_{1}\right)=v_{2}$.

Therefore, in all cases, if $f_{\#}=f_{s}\left(r, 1, c_{1}, c_{2}\right)$ then

$$
\bar{\nu}^{-1} \circ(1, f)_{\#}: \pi_{1}\left(M(\phi),\left\langle x_{2}, 0\right\rangle\right) \rightarrow \pi_{1}\left(M(\phi) \times_{S^{1}} M(\phi),\left(\left\langle x_{2}, 0\right\rangle,\left\langle x_{1}, 0\right\rangle\right)\right)
$$

is given by $\alpha \mapsto \alpha_{1} u_{1}^{r}, \beta \mapsto \beta_{1} u_{1}^{s} v_{1}$ and $c_{0} \mapsto u_{1}^{c_{1}} v_{1}^{c_{2}} c_{01}$.

The next theorem describes the homomorphisms of diagram (1.1).

TheOREM 5.1. Let $f_{s}\left(r, 1, c_{1}, c_{2}\right)$ be one of the four cases given by Theorem $2.4, \alpha, \beta, c_{0}$ loops in $M(\phi)$ based at $\left\langle x_{2}, 0\right\rangle$ given respectively by $\alpha=$ $\left\langle\varrho_{21} \varrho_{22}, 0\right\rangle, \beta=\left\langle\varrho_{22}^{-1}, 0\right\rangle, c_{0}(t)=\left\langle x_{2}, t\right\rangle$, and $\bar{a}=\varrho_{11} \varrho_{12}, \bar{b}=\varrho_{12}^{-1}$ loops in $K-x_{2}$ based at $x_{1}$. Then:

(1) The homomorphism

$(1, f)_{\#}: \pi_{1}\left(M(\phi),\left\langle x_{2}, 0\right\rangle\right) \rightarrow \pi_{1}\left(M(\phi) \times_{S^{1}} M(\phi),\left(\left\langle x_{2}, 0\right\rangle,\left\langle x_{2}, 0\right\rangle\right)\right)$ is given by

$$
\alpha \mapsto \alpha_{2} u_{2}^{r}, \quad \beta \mapsto \beta_{2} u_{2}^{s} v_{2}, \quad c_{0} \mapsto u_{2}^{c_{1}} v_{2}^{c_{2}} c_{02} .
$$

(2) The homomorphism $\bar{\nu}$ is given by $\bar{\nu}(\eta)=\nu^{-1} \eta \nu$ where $\nu: I \rightarrow$ $M(\phi) \times_{S^{1}} M(\phi)$ is given by $\nu(t)=\left(\left\langle x_{2}, 0\right\rangle,\langle\sigma(t), 0\rangle\right)$ with $\sigma: I \rightarrow K$ a path joining $x_{1}$ to $x_{2}$, and the homomorphism $\kappa$ is given by $\tilde{\alpha} \mapsto \alpha_{1}$, $\tilde{\beta} \mapsto \beta_{1}, \tilde{c}_{0} \mapsto c_{01}, \tilde{a} \mapsto u_{1}$ and $\tilde{b} \mapsto v_{1}$.

(3) The lifting homomorphism $\psi: \pi_{1}\left(M(\phi),\left\langle x_{2}, 0\right\rangle\right) \rightarrow \pi_{1}\left(M(\phi) \times_{S^{1}} M(\phi)-\Delta,\left(\left\langle x_{2}, 0\right\rangle,\left\langle x_{1}, 0\right\rangle\right)\right)$ exists if and only if we can find elements $Z_{1}, Z_{2}, Z_{3} \in \pi_{2}(K$, $\left.K-x_{2}, x_{1}\right)$ and $A, F, C \in \pi_{1}\left(M(\phi) \times_{S^{1}} M(\phi)-\Delta,\left(\left\langle x_{2}, 0\right\rangle,\left\langle x_{1}, 0\right\rangle\right)\right)$ 
such that

$$
\psi: \begin{cases}\alpha \mapsto Z_{1} A & \text { with } \kappa(A)=\alpha_{1} u_{1}^{r} \text { if } f_{\#}(\alpha)=\alpha^{r}, \\ \beta \mapsto Z_{2} F & \text { with } \kappa(F)=\beta_{1} u_{1}^{s} v_{1} \text { if } f_{\#}(\beta)=\alpha^{s} \beta, \\ c_{0} \mapsto Z_{3} C & \text { with } \kappa(C)=u_{1}^{c_{1}} v_{1}^{c_{2}} c_{01} \text { if } f_{\#}\left(c_{0}\right)=\alpha^{c_{1}} \beta^{c_{2}} c_{0},\end{cases}
$$

and it must satisfy $\psi\left(\alpha \beta \alpha \beta^{-1}\right)=1, \psi\left(c_{0} \alpha c_{0}^{-1} \alpha^{-\epsilon}\right)=1$ and $\psi\left(c_{0} \beta c_{0}^{-1} \beta^{-\eta} \alpha^{-p}\right)=1$ where $p \in\{0,1\}, \epsilon= \pm 1$ and $\eta= \pm 1$.

Proof. (1) and (2) follow from the considerations before.

Therefore it is enough to prove (3). First we observe that if $\psi(\alpha)=x$ and $f_{\#}(\alpha)=\alpha^{r}$ then $\kappa(x)=\bar{\nu}^{-1} \circ(1, f)_{\#}(\alpha)=\alpha_{1} u_{1}^{r}$. On the other hand, if $\kappa(A)=\alpha_{1} u_{1}^{r}$ then $x A^{-1}=Z_{1}$, where $Z_{1} \in \pi_{2}\left(K, K-x_{2}, x_{1}\right)=$ kernel of $\pi_{1}\left(K-x_{2}, x_{1}\right) \rightarrow \pi_{1}\left(K, x_{1}\right)$, and the result follows. Similarly we argue for $\beta$ and $c_{0}$. Now the equalities $\psi\left(\alpha \beta \alpha \beta^{-1}\right)=1, \psi\left(c_{0} \alpha c_{0}^{-1} \alpha^{-\epsilon}\right)=1$ and $\psi\left(c_{0} \beta c_{0}^{-1} \beta^{-\eta} \alpha^{-p}\right)=1$ follow from the relations in $\pi_{1}\left(M(\phi),\left\langle x_{2}, 0\right\rangle\right)$.

Now we will derive the equations that $Z_{1}, Z_{2}, Z_{3}, A, F, C$ must satisfy in order that there exists a lifting

$$
\psi: \pi_{1}\left(M(\phi),\left\langle x_{2}, 0\right\rangle\right) \rightarrow \pi_{1}\left(M(\phi) \times_{S^{1}} M(\phi)-\Delta,\left(\left\langle x_{2}, 0\right\rangle,\left\langle x_{1}, 0\right\rangle\right)\right)
$$

in each of the four cases given by Theorem 2.4.

From the theorem above, for $f_{\#}=f_{s}\left(r, 1, c_{1}, c_{2}\right), \psi$ is of the form

$$
\begin{cases}\alpha \mapsto Z_{1} A & \text { with } \kappa(A)=\alpha_{1} u_{1}^{r} \\ \beta \mapsto Z_{2} F & \text { with } \kappa(F)=\beta_{1} u_{1}^{s} v_{1} \\ c_{0} \mapsto Z_{3} C & \text { with } \kappa(C)=u_{1}^{c_{1}} v_{1}^{c_{2}} c_{01}\end{cases}
$$

and it must satisfy $\psi\left(\alpha \beta \alpha \beta^{-1}\right)=1, \psi\left(c_{0} \alpha c_{0}^{-1} \alpha^{-\epsilon}\right)=1$ and $\psi\left(c_{0} \beta c_{0}^{-1} \beta^{-\eta} \alpha^{-p}\right)$ $=1$ where $p \in\{0,1\}, \epsilon= \pm 1$ and $\eta= \pm 1$. Hence, there exists a lift $\psi$ if and only if the system of equations

$$
\left\{\begin{array}{c}
Z_{1}\left(A Z_{2} A^{-1}\right)\left(A F A F^{-1}\right)\left(F A^{-1} Z_{1} A F^{-1}\right) Z_{2}^{-1}=1 \\
Z_{3}\left(C Z_{1} C^{-1}\right)\left(C A C^{-1} A^{-1}\right)\left(A Z_{3}^{-1} A^{-1}\right) Z_{1}^{-1}=1 \\
Z_{3}\left(C Z_{2} C^{-1}\right)\left(C F C^{-1} F^{-\eta} A^{-p}\right)\left(A^{p} F^{\eta} Z_{3}^{-1} F^{-\eta} A^{-p}\right) \\
\cdot\left(A^{p} F^{(\eta-1) / 2} Z_{2}^{-\eta} F^{(1-\eta) / 2} A^{-p}\right) Z_{1}^{-p}=1
\end{array}\right.
$$

has a solution in $Z_{1}, Z_{2}, Z_{3}$.

We refer to $(I)$ as the system generated by the input data $(A, F, C ; r, s$, $\left.\left(c_{1}, c_{2}\right)\right)$ where $\kappa(A)=\alpha_{1} u_{1}^{r}, \kappa(F)=\beta_{1} u_{1}^{s} v_{1}$ and $\kappa(C)=u_{1}^{c_{1}} v_{1}^{c_{2}} c_{01}$.

Each equation in $(I)$ involves a product where all the factors are conjugates of $Z_{i}$, except one. The factor which is not a conjugate of $Z_{i}$ is called the constant term of the equation; these are $A F A F^{-1}, C A C^{-1} A^{-1}$ and $C F C^{-1} F^{-\eta} A^{-p}$, respectively. Since the variables $Z_{j}$ belong to the normal 
subgroup $\pi_{2}\left(K, K-x_{2}, x_{1}\right)$ of $\pi_{1}\left(M(\phi) \times_{S^{1}} M(\phi)-\Delta,\left(\left\langle x_{2}, 0\right\rangle,\left\langle x_{1}, 0\right\rangle\right)\right)$, all factors (including the constant terms) belong to $\pi_{2}\left(K, K-x_{2}, x_{1}\right)$.

Proposition 5.2. There is a solution of the system generated by the input data $\left(A_{1}, F_{1}, C_{1} ; r, s,\left(c_{1}, c_{2}\right)\right)$ if and only if there exist solutions for all systems generated by any input data $\left(A, F, C ; r, s,\left(c_{1}, c_{2}\right)\right)$.

Proof. Suppose that $X_{1}, X_{2}, X_{3}$ is a solution for the system generated by $\left(A_{1}, F_{1}, C_{1} ; r, s,\left(c_{1}, c_{2}\right)\right)$. As $\kappa\left(A_{1}\right)=\kappa(A), \kappa\left(F_{1}\right)=\kappa(F)$ and $\kappa\left(C_{1}\right)=\kappa(C)$ we can write $A_{1}=Y_{1} A, F_{1}=Y_{2} F$ and $C_{1}=Y_{3} C$ for some $Y_{j} \in \pi_{2}(K$, $\left.K-x_{2}, x_{1}\right)$. When we substitute these in the above system we obtain

$$
\left\{\begin{array}{l}
X_{1} Y_{1} A X_{2} Y_{2} F X_{1} Y_{1} A F^{-1} Y_{2}^{-1} X_{2}^{-1}=1 \\
X_{3} Y_{3} C X_{1} Y_{1} A C^{-1} Y_{3}^{-1} X_{3}^{-1} A^{-1} Y_{1}^{-1} X_{1}^{-1}=1 \\
X_{3} Y_{3} C X_{2} Y_{2} F C^{-1} Y_{3}^{-1} X_{3}^{-1}\left(F^{-1} Y_{2}^{-1} X_{2}^{-1}\right)^{\eta}\left(A^{-1} Y_{1}^{-1} X_{1}^{-1}\right)^{p}=1 .
\end{array}\right.
$$

Therefore $Z_{1}=X_{1} Y_{1}, Z_{2}=X_{2} Y_{2}, Z_{3}=X_{3} Y_{3}$ is a solution of the system generated by the input data $\left(A, F, C ; r, s,\left(c_{1}, c_{2}\right)\right)$. The converse is immediate.

Conjugating the equations of the system by a word $q$ in $\tilde{a}$ and $\tilde{b}$ (or $v$ and $w$ ) we find that $\kappa(q)=u_{1}^{m} v_{1}^{n}$ and so we obtain

THEOREM 5.3. Let $\phi=\phi_{p}(1, \eta)$ be one of the four cases given by Theorem 2.4. If $q$ denotes a word so that $\kappa(q)=u_{1}^{m} v_{1}^{n}$ then conjugating by $q$ the equations of the system $(I)$ generated by the input data $\left(A, F, C ; r, s,\left(c_{1}, c_{2}\right)\right)$, we obtain a new system generated by

$$
\begin{aligned}
& \left(A^{\prime}, F^{\prime}, C^{\prime} ;(-1)^{n} r, 2 m+(-1)^{n} s\right. \\
& \left.\left(\left(1-(-1)^{c_{2}+(1-\eta) n}\right) m+(-1)^{c_{2}+n}\left(\frac{1-(-1)^{n}}{2}\right) p+(-1)^{n} c_{1},(1-\eta) n+c_{2}\right)\right)
\end{aligned}
$$

where $A^{\prime}=q A q^{-1}, F^{\prime}=q F q^{-1}$ and $C^{\prime}=q C q^{-1}$. Moreover, if $\left(Z_{1}, Z_{2}, Z_{3}\right)$ is a solution of the system $(I)$ then $\left(Z_{1}^{\prime}=q Z_{1} q^{-1}, Z_{2}^{\prime}=q Z_{2} q^{-1}, Z_{3}^{\prime}=\right.$ $\left.q Z_{3} q^{-1}\right)$ is a solution of the new system given above.

Proof. The final part is straightforward. For the first part it is sufficient to calculate $\kappa\left(A^{\prime}\right), \kappa\left(F^{\prime}\right)$ and $\kappa\left(C^{\prime}\right)$.

Using the relations in $\pi_{1}\left(M(\phi) \times_{S^{1}} M(\phi),\left(\left\langle x_{2}, 0\right\rangle,\left\langle x_{1}, 0\right\rangle\right)\right)$ we obtain

$$
\begin{aligned}
\kappa\left(A^{\prime}\right) & =\kappa\left(q A q^{-1}\right)=u_{1}^{m} v_{1}^{n} \alpha_{1} u_{1}^{r} v_{1}^{-n} u_{1}^{-m}=\alpha_{1} \alpha_{1}^{-1} u_{1}^{m} v_{1}^{n} \alpha_{1} u_{1}^{r} v_{1}^{-n} u_{1}^{-m} \\
& =\alpha_{1} u_{1}^{m} v_{1}^{n} u_{1}^{r} v_{1}^{-n} u_{1}^{-m}=\alpha_{1} u_{1}^{m} u_{1}^{(-1)^{n} r} v_{1}^{n} v_{1}^{-n} u_{1}^{-m}=\alpha_{1} u_{1}^{(-1)^{n} r}
\end{aligned}
$$

Similarly $\kappa\left(F^{\prime}\right)=\beta_{1} u_{1}^{2 m+(-1)^{n} s} v_{1}$. 
For the system $(I)$ we have

$$
\begin{aligned}
& \kappa\left(C^{\prime}\right)=\kappa\left(q C q^{-1}\right)=u_{1}^{m} v_{1}^{n} u_{1}^{c_{1}} v_{1}^{c_{2}} c_{01} v_{1}^{-n} u_{1}^{-m} \\
& =u_{1}^{m} v_{1}^{n} u_{1}^{c_{1}} v_{1}^{c_{2}} c_{01} v_{1}^{-n} u_{1}^{-m} c_{01}^{-1} c_{01}
\end{aligned}
$$

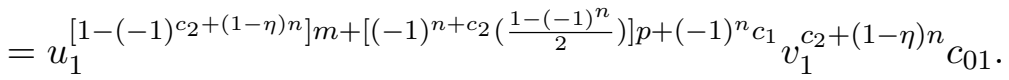

REMARK 5.1. As a consequence of the above theorem the existence of a lifting for $f_{s}\left(r, 1, c_{1}, 2 k\right)$ or $f_{s}(r, 1, s, 2 k+1)$ is equivalent to the existence of a lifting for $f_{2 m+(-1)^{n} s}\left((-1)^{n} r, 1,-\left(\frac{1-(-1)^{n}}{2}\right) p+(-1)^{n} c_{1},(1-\eta) n+2 k\right)$ or $f_{2 m+(-1)^{n} s}\left((-1)^{n} r, 1,2 m+\left(\frac{1-(-1)^{n}}{2}\right) p+(-1)^{n} s,(1-\eta) n+2 k+1\right)$ respectively.

Using the results above we can reduce the cases to be analyzed.

COROLlary 5.4. In order to study the problem of existence of solution of the system generated by the input data $\left(A, F, C ; r, s,\left(c_{1}, c_{2}\right)\right)$ it suffices to solve the problem for input data given by the homomorphisms $f_{s}\left(r, 1, c_{1}, c_{2}\right)$ listed in the following table:

\begin{tabular}{|c|l|}
\hline Case I & I.1: $f_{s}(r, 1,0,2 k): \alpha \mapsto \alpha^{r}, \beta \mapsto \alpha^{s} \beta, c_{0} \mapsto \beta^{2 k} c_{0}, r \geq 0, s \in\{0,1\}, k \in \mathbb{Z}$ \\
$\phi_{0}(1,1)$ & I.2: $f_{s}(0,1, s, 2 k+1): \alpha \mapsto 1, \beta \mapsto \alpha^{s} \beta, c_{0} \mapsto \alpha^{s} \beta^{2 k+1} c_{0}, s \in\{0,1\}, k \in \mathbb{Z}$ \\
\hline Case II & $f_{s}(2 r+1,1, r, 2 k): \alpha \mapsto \alpha^{2 r+1}, \beta \mapsto \alpha^{s} \beta, c_{0} \mapsto \alpha^{r} \beta^{2 k} c_{0}, r \geq 0, s \in\{0,1\}, k \in \mathbb{Z}$ \\
$\phi_{1}(1,1)$ & \\
\hline Case III & III.1: $f_{s}(r, 1,0,2 k): \alpha \mapsto \alpha^{r}, \beta \mapsto \alpha^{s} \beta, c_{0} \mapsto \beta^{2 k} c_{0}, r \geq 0, s \in\{0,1\}, k \in\{0,1\}$ \\
$\phi_{0}(1,-1)$ & III.2: $f_{s}(0,1, s, 1): \alpha \mapsto 1, \beta \mapsto \alpha^{s} \beta, c_{0} \mapsto \alpha^{s} \beta c_{0}, s \in\{0,1\}$ \\
\hline Case IV & $f_{s}(2 r+1,1, r, 0): \alpha \mapsto \alpha^{2 r+1}, \beta \mapsto \alpha^{s} \beta, c_{0} \mapsto \alpha^{r} c_{0}, r \in \mathbb{Z}, s \in\{0,1\}$ \\
$\phi_{1}(1,-1)$ & \\
\hline
\end{tabular}

Proof. Let $\mathcal{S}$ be the system generated by $\left(A, F, C ; r, s,\left(c_{1}, c_{2}\right)\right)$ where $f_{s}\left(r, 1, c_{1}, c_{2}\right)$ is given by the table of Theorem 2.4. From Theorem 5.3, by straightforward calculation in each of the four cases, it follows that there is a system $\mathcal{S}^{\prime}$, generated by $\left(A^{\prime}, F^{\prime}, C^{\prime} ; r^{\prime}, s^{\prime},\left(c_{1}^{\prime}, c_{2}^{\prime}\right)\right)$ where $f_{s^{\prime}}\left(r^{\prime}, 1, c_{1}^{\prime}, c_{2}^{\prime}\right)$ belongs to the table in the statement of the corollary, such that $\mathcal{S}$ has a solution if and only if $\mathcal{S}^{\prime}$ has a solution.

Now from Proposition 5.2 there is a solution for $\mathcal{S}^{\prime}$ if and only if there exist solutions for all systems $\mathcal{S}^{\prime \prime}$ determined by $f_{s^{\prime}}\left(r^{\prime}, 1, c_{1}^{\prime}, c_{2}^{\prime}\right)$. So the result follows.

6. The systems on some quotients and the main classification theorem. The system $(I)$ given after Theorem 5.1 is on $\pi_{2}\left(K, K-x_{2}, x_{1}\right)=$ $\pi_{2}$. Now, we will look at the equations of that system on some quotients of the abelianized $\pi_{2}$, which is $\left(\pi_{2}\right)_{\mathrm{ab}}=\pi_{2} /\left[\pi_{2}, \pi_{2}\right]$. Whenever one of those equations on the abelianization $\left(\pi_{2}\right)$ ab has no solution, we can infer that the 
initial system has no solution. On the other hand, if the system has a solution on the abelianization we will try to find a solution on $\pi_{2}$ itself. In order to decide whether the equations on the abelianization $\left(\pi_{2}\right)_{\text {ab }}$ have no solution, we project the system to $\mathbb{Z}$ using the augmentation homomorphism $\mathcal{E}$ : $\left(\pi_{2}\right)_{\mathrm{ab}} \rightarrow \mathbb{Z}$ and study if the corresponding equation on $\mathbb{Z}$ has a solution or not. We will show that one system has a solution if and only if the other does.

We recall that the group $\pi_{1}\left(K, x_{1}\right)$ acts on $\pi_{2} /\left[\pi_{2}, \pi_{2}\right]=\left(\pi_{2}\right)$ ab since for each $\xi \in \pi_{1}\left(K-x_{2}, x_{1}\right)$ we have the commutative diagram

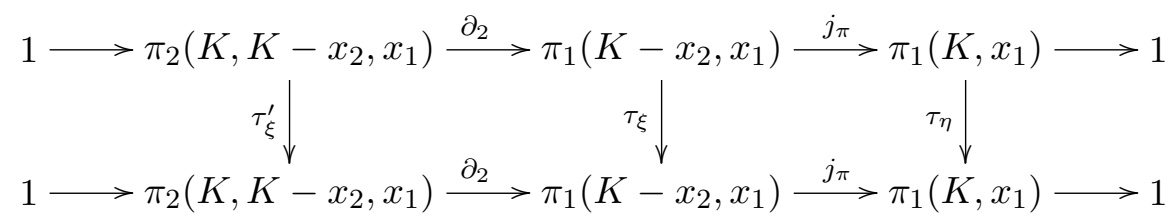

where $\eta=j_{\pi}(\xi)$.

We note that $\pi_{2}$ is the kernel of the map $j_{\pi}:\langle\bar{w}, \bar{v}\rangle=\pi_{1}\left(K-x_{2}, x_{1}\right) \rightarrow$ $\pi_{1}\left(K, x_{1}\right)=\left\langle\bar{w}, \bar{v} \mid \bar{w}^{-1} \bar{v}^{-1} \bar{w}^{-1} \bar{v}=1\right\rangle$ and we set $B=w^{-1} v^{-1} w^{-1} v$ where $w, v$ are as in Remark 4.1.

THEOREM 6.1.

(1) There is an isomorphism $\left(\pi_{2}\right)_{\mathrm{ab}} \cong \mathbb{Z}\left[\pi_{1}(K)\right]$, where $\mathbb{Z}\left[\pi_{1}(K)\right]$ is the group ring of the fundamental group of the Klein bottle.

(2) The abelianization homomorphism $\mathcal{A}: \pi_{2} \rightarrow\left(\pi_{2}\right)_{\text {ab }}$ sends the element $p(w, v) B(p(w, v))^{-1}$ to $1 .[p(\bar{w}, \bar{v})]$ where $[p(\bar{w}, \bar{v})]=j_{\pi}(p(\bar{w}, \bar{v}))$.

Proof. This follows from [Ly50, end of page 650].

Let $\mathcal{A}: \pi_{2}\left(K, K-x_{2}, x_{1}\right)=\pi_{2} \rightarrow \pi_{2} /\left[\pi_{2}, \pi_{2}\right]=\left(\pi_{2}\right)_{\text {ab }}$ be the projection. Then $\mathcal{A}\left(p(w, v) B^{t}(p(w, v))^{-1}\right)=t .\left(\bar{w}^{x} \bar{v}^{y}\right)$ where $p(w, v) B^{t} p(w, v)^{-1}=$ $j_{2 \#}\left(p(\bar{w}, \bar{v}) \bar{E}^{t}(p(\bar{w}, \bar{v}))^{-1}\right)$ and $j_{\pi}(p(\bar{w}, \bar{v}))=\bar{w}^{x} \bar{v}^{y}$ for some $x, y \in \mathbb{Z}$, where $p(w, v)$ and $p(\bar{w}, \bar{v})$ denote the same words in $w, v$ and $\bar{w}, \bar{v}$ respectively. Now we define the $\pi_{1}\left(K, x_{1}\right)$-homomorphism $\mathcal{E}:\left(\pi_{2}\right)_{\mathrm{ab}} \rightarrow \mathbb{Z}$ by $\mathcal{E}\left(t \cdot\left(\bar{w}^{x} \bar{v}^{y}\right)\right)=t$.

In the first subsection we study the system on the abelianization and in the second subsection we prove the classification result.

6.1. The system of equations on quotients of $\left(\pi_{2}\right)_{\mathrm{ab}} \cong \mathbb{Z}\left[\pi_{1}(K)\right]$. In this subsection we will calculate $A F A F^{-1}, \mathcal{E} \circ \mathcal{A}\left(C A C^{-1} A^{-1}\right)$ and $\mathcal{E} \circ$ $\mathcal{A}\left(C F C^{-1} F A^{-p}\right), p=0,1$, where $A F A F^{-1}, C A C^{-1} A^{-1}$ and $C F C^{-1} F A^{-p}$ are the constant terms of the equations of the system. Then we apply $\mathcal{E} \circ \mathcal{A}$ to the equations. In certain cases we project the system onto another quotient.

If $f_{s}\left(r, 1, c_{1}, c_{2}\right)$ is the homomorphism on $\pi_{1}(M(\phi(1, \eta)))$ defined before, then the terms appearing in the system are denoted by $A=\tilde{\alpha} w^{r}, r \in \mathbb{Z}$, $F=\tilde{\beta} v w^{1-s}, s \in\{0,1\}$, and $C=w^{c_{1}}(v w)^{c_{2}} \tilde{c}_{0}$.

Because $w=\tilde{a} \tilde{b} \tilde{a}^{-1} \tilde{b}^{-1} \tilde{a}^{-1}$ and $v=\tilde{a} \tilde{b}$ we have $\kappa(v)=\kappa(\tilde{a} \tilde{b})=u_{1} v_{1}$, $\kappa(w)=\kappa\left(\tilde{a} \tilde{b} \tilde{a}^{-1} \tilde{b}^{-1} \tilde{a}^{-1}\right)=u_{1}\left(v_{1} u_{1}^{-1} v_{1}^{-1} u_{1}^{-1}\right)=u_{1}$ and therefore $\kappa\left(\tilde{\alpha} \tilde{a}^{r}\right)=$ 
$\kappa\left(\tilde{\alpha} w^{r}\right)=\kappa(A), \kappa\left(\tilde{\beta} \tilde{a}^{s} \tilde{b}\right)=\kappa\left(\tilde{\beta} v w^{1-s}\right)=\kappa(F)$ and $\kappa(C)=\kappa\left(w^{c_{1}}(v w)^{c_{2}} \tilde{c}_{0}\right)$ $=u_{1}^{c_{1}}\left(u_{1} v_{1} u_{1}\right)^{c_{2}} c_{01}=u_{1}^{c_{1}} v_{1}^{c_{2}} c_{01}=\kappa\left(\tilde{a}^{c_{1}} \tilde{b}^{c_{2}} \tilde{c}_{0}\right)$.

Proposition 6.2. If $A=\tilde{\alpha} w^{r}, F=\tilde{\beta} v w^{1-s}$ where $r \in \mathbb{Z}$ and $s \in\{0,1\}$ then $A F A F^{-1}=1$. Hence $\mathcal{E} \circ \mathcal{A}\left(A F A F^{-1}\right)=0$.

Proof. We have

$$
\begin{aligned}
A F A F^{-1} & =\tilde{\alpha} w^{r} \tilde{\beta} v w^{1-s} \tilde{\alpha} w^{r} w^{s-1} v^{-1} \tilde{\beta}^{-1} \\
& =\tilde{\alpha} w^{r} \tilde{\alpha}^{-1} \tilde{\alpha} \tilde{\beta} v w^{1-s} \tilde{\beta}^{-1} \tilde{\alpha}^{-1}\left(\tilde{\alpha} \tilde{\beta} \tilde{\alpha} \tilde{\beta}^{-1}\right) \tilde{\beta} w^{r+s-1} v^{-1} \tilde{\beta}^{-1} \\
& =w^{r+s} v w B v^{-1} w^{-r-s+1}=w^{r+s-1} w^{-r-s+1}=1 .
\end{aligned}
$$

The second part is clear.

Now, let us consider the term $C A C^{-1} A^{-1}$. If we let $a=\frac{-1+\eta}{2}$ then we have

$$
\begin{aligned}
C A C^{-1} A^{-1}= & w^{c_{1}}(v w)^{c_{2}} \tilde{c}_{0} \tilde{\alpha} w^{r} \tilde{c}_{0}^{-1}(v w)^{-c_{2}} w^{-c_{1}} w^{-r} \tilde{\alpha}^{-1} \\
= & w^{c_{1}}(v w)^{c_{2}} B^{a} \tilde{\alpha} v^{a} w^{\eta r} v^{-a}(v w)^{-c_{2}} w^{-c_{1}} w^{-r} \tilde{\alpha}^{-1} \\
= & \left(w^{c_{1}}(v w)^{c_{2}} B^{a}(v w)^{-c_{2}} w^{-c_{1}}\right) \\
& \cdot\left(w^{c_{1}}(v w)^{c_{2}}(w v w)^{a} w^{\eta r}(w v w)^{-a}(w v w w)^{-c_{2}} w^{-r} w^{-c_{1}}\right) .
\end{aligned}
$$

In Cases I and II we have $\eta=1$ and therefore $a=0$. So

$$
C A C^{-1} A^{-1}=w^{c_{1}}\left[(v w)^{c_{2}} w^{r}(w v w w)^{-c_{2}} w^{-r}\right] w^{-c_{1}} .
$$

Proposition 6.3. If $\eta=1$ then $\mathcal{E} \circ \mathcal{A}\left(C A C^{-1} A^{-1}\right)=c_{2}$. Hence:

(1) In Cases I.1 and II, $\mathcal{E} \circ \mathcal{A}\left(C A C^{-1} A^{-1}\right)=2 k, k \in \mathbb{Z}$.

(2) In Case I.2, $\mathcal{E} \circ \mathcal{A}\left(C A C^{-1} A^{-1}\right)=2 k+1, k \in \mathbb{Z}$.

Proof. Consider $C A C^{-1} A^{-1}$ for $r=0$, which is $w^{c_{1}}\left[(v w)^{c_{2}}\left(w^{-1} B v^{-1}\right)^{c_{2}}\right]$ $\cdot w^{-c_{1}}$. We will show that $\mathcal{E} \circ \mathcal{A}\left((v w)^{c_{2}}\left(w^{-1} B v^{-1}\right)^{c_{2}}\right)=c_{2}$ for $c_{2} \in \mathbb{Z}$, which implies the assertion for Case I.2.

If $c_{2}=1$ then $(v w)^{c_{2}}\left(w^{-1} B v^{-1}\right)^{c_{2}}=v w w^{-1} B v^{-1}=v B v^{-1}$ and therefore $\mathcal{E} \circ \mathcal{A}\left(v B v^{-1}\right)=1$. Suppose that the assertion is true for $c_{2}-1$. Then

$$
\begin{aligned}
(v w)^{c_{2}}\left(w^{-1} B v^{-1}\right)^{c_{2}} & =(v w)^{c_{2}-1}(v w)\left(w^{-1} B v^{-1}\right)\left(w^{-1} B v^{-1}\right)^{c_{2}-1} \\
& =(v w)^{c_{2}-1}\left(v B v^{-1}\right)\left(w^{-1} B v^{-1}\right)^{c_{2}-1} \\
& =\left[(v w)^{c_{2}-1}\left(v B v^{-1}\right)(v w)^{1-c_{2}}\right](v w)^{c_{2}-1}\left(w^{-1} B v^{-1}\right)^{c_{2}-1} .
\end{aligned}
$$

Therefore

$$
\begin{aligned}
\mathcal{E} \circ \mathcal{A}\left((v w)^{c_{2}}\left(w^{-1} B v^{-1}\right)^{c_{2}}\right)= & \mathcal{E} \circ \mathcal{A}\left((v w)^{c_{2}-1}\left(v B v^{-1}\right)(v w)^{1-c_{2}}\right) \\
& +\mathcal{E} \circ \mathcal{A}\left((v w)^{c_{2}-1}\left(w^{-1} B v^{-1}\right)^{c_{2}-1}\right) \\
= & 1+\left(c_{2}-1\right)=c_{2} .
\end{aligned}
$$

The proof for Cases I.1 and II follows from the two lemmas below. 
Lemma 6.4. $\mathcal{E} \circ \mathcal{A}\left(\left[v^{2}, w^{r}\right]\right)=0$ for any $r \geq 0$.

Proof. The case $r=0$ is trivial. The rest of the proof is straightforward induction on $r$.

Lemma 6.5. If $c_{2}$ is even then $\mathcal{E} \circ \mathcal{A}\left((v w)^{c_{2}} w^{r}\left(w^{-1} B v^{-1}\right)^{c_{2}} w^{-r}\right)=c_{2}$.

Proof. The case $c_{2}=0$ is trivial. If $c_{2}=2$ the conclusion follows by straightforward calculation using Lemma 6.4. For the general case we use induction on $c_{2}$.

In Cases III and IV we have $\eta=-1$ and therefore $a=-1$. So

$$
\begin{aligned}
C A C^{-1} A^{-1}= & \left(w^{c_{1}}(v w)^{c_{2}} B^{-1}(v w)^{-c_{2}} w^{-c_{1}}\right) \\
& \cdot\left(w^{c_{1}}(v w)^{c_{2}}(w v w)^{-1} w^{-r}(w v w)(w v w w)^{-c_{2}} w^{-r} w^{-c_{1}}\right) .
\end{aligned}
$$

Proposition 6.6. If $\eta=-1$ then $\mathcal{E} \circ \mathcal{A}\left(C A C^{-1} A^{-1}\right)=c_{2}+r-1$. Hence:

(1) In Case III.1, $\mathcal{E} \circ \mathcal{A}\left(C A C^{-1} A^{-1}\right)=2 k+r-1, r \geq 0, k \in\{0,1\}$.

(2) In Case III.2, $\mathcal{E} \circ \mathcal{A}\left(C A C^{-1} A^{-1}\right)=0$.

(3) In Case IV, $\mathcal{E} \circ \mathcal{A}\left(C A C^{-1} A^{-1}\right)=r-1, r \in \mathbb{Z}$.

Proof. Consider $C A C^{-1} A^{-1}$ in Case III.2, where $c_{2}=1, c_{1}=s$ and $r=0$. So we have

$$
\begin{aligned}
C A C^{-1} A^{-1}= & \left(w^{s}(v w) B^{-1}(v w)^{-1} w^{-s}\right) \\
& \cdot\left(w^{s}(v w)(w v w)^{-1}(w v w)(w v w w)^{-1} w^{-s}\right) \\
= & \left(w^{s}(v w) B^{-1}(v w)^{-1} w^{-s}\right)\left(w^{s} v B v^{-1} w^{-s}\right),
\end{aligned}
$$

and therefore $\mathcal{E} \circ \mathcal{A}\left(C A C^{-1} A^{-1}\right)=-1+1=0$.

The proof for Cases III.1 and IV follows from the two lemmas below.

LEMMA 6.7. $\mathcal{E} \circ \mathcal{A}\left(v^{-1} w^{-r} v w^{-r}\right)=r$ and $\mathcal{E} \circ \mathcal{A}\left((v w)^{-1} w^{-r}(v w) w^{-r}\right)$ $=r$ for $r \in \mathbb{Z}$.

Proof. For the first part assume that $r \geq 0$. The case $r \leq 0$ is similar and left to the reader.

If $r=0$ then the assertion is trivial. The rest of the proof is induction on $r$.

LEMMA 6.8. If $c_{2}$ is even then

$$
\mathcal{E} \circ \mathcal{A}\left(C A C^{-1} A^{-1}\right)=-1+\mathcal{E} \circ \mathcal{A}\left((v w)^{-1} w^{-r} v w w^{-r}\right)+c_{2} .
$$

Proof. We have

$$
\begin{aligned}
C A C^{-1} A^{-1}= & \left(w^{c_{1}}(v w)^{c_{2}} B^{-1}(v w)^{-c_{2}} w^{-c_{1}}\right) \\
& \cdot\left(w^{c_{1}}(v w)^{c_{2}}(w v w)^{-1} w^{-r}(w v w)(w v w w)^{-c_{2}} w^{-r} w^{-c_{1}}\right) .
\end{aligned}
$$

Using Lemma 6.5 we obtain the result. 
Now consider the term $C F C^{-1} F A^{-p}$. We compute $\mathcal{E} \circ \mathcal{A}\left(C F C^{-1} F A^{-p}\right)$ in Cases III and IV. Because we will show that the necessary conditions obtained so far for Cases I and II are also sufficient, we do not need to compute $\mathcal{E} \circ \mathcal{A}\left(C F C^{-1} F A^{-p}\right)$ in Cases I and II.

Proposition 6.9. In Case III, where $p=0$, we have

$$
\mathcal{E} \circ \mathcal{A}\left(C F C^{-1} F\right)= \begin{cases}-2 k+s-1 & \text { in Case III.1, } \\ -2, & \text { in Case III.2. }\end{cases}
$$

Proof. In Case III.1 we have $F=\tilde{\beta} v w^{1-s}$ with $s \in\{0,1\}$ and $C=$ $(v w)^{2 k} \tilde{c}_{0}$ with $k \in\{0,1\}$. So $C F C^{-1} F=(v w)^{2 k} v^{-1} w^{1-s} v\left(v w^{-1}\right)^{-2 k} w^{1-s}$.

If $k=0$ and $s=0$ then $C F C^{-1} F=v^{-1} w v w=B^{-1}$ and it follows that $\mathcal{E} \circ \mathcal{A}\left(C F C^{-1} F\right)=-1$.

If $k=0$ and $s=1$ then $C F C^{-1} F=v^{-1} v=1$ and hence $\mathcal{E} \circ \mathcal{A}\left(C F C^{-1} F\right)$ $=0$.

If $k=1$ and $s=0$ then

$$
C F C^{-1} F=\left[(v w)^{2} B^{-1}(v w)^{-2}\right]\left(v^{2} B^{-1} v^{-2}\right)\left(w^{-1} v B^{-1} v^{-1} w\right)
$$

and so $\mathcal{E} \circ \mathcal{A}\left(C F C^{-1} F\right)=-3$.

If $k=1$ and $s=1$ then $C F C^{-1} F=\left(v^{2} B^{-1} v^{-2}\right)\left(v^{2} w B^{-1} w^{-1} v^{-2}\right)$ and therefore $\mathcal{E} \circ \mathcal{A}\left(C F C^{-1} F\right)=-2$.

In Case III.2 we have $F=\tilde{\beta} v w^{1-s}$ and $C=w^{s} v w \tilde{c}_{0}$ where $s \in\{0,1\}$. So $C F C^{-1} F=\left(w^{s-1} v B^{-1} v^{-1} w^{1-s}\right)\left(w^{-1} v B^{-1} v^{-1} w\right)$ and the result follows.

Now we consider Case IV. Then $A=\tilde{\alpha} w^{2 r+1}, F=\tilde{\beta} v w^{1-s}$ and $C=w^{r} \tilde{c}_{0}$ where $r \in \mathbb{Z}$ and $s \in\{0,1\}$.

Proposition 6.10. In Case IV we have $\mathcal{E} \circ \mathcal{A}\left(C F C^{-1} F A^{-1}\right)=s-1$ where $F=\tilde{\beta} v w^{1-s}, s \in\{0,1\}$.

Proof. We have

$$
\begin{aligned}
C F C^{-1} F A^{-1} & =w^{r} \tilde{c}_{0} \tilde{\beta} v w^{1-s} \tilde{c}_{0}^{-1} w^{-r} \tilde{\beta} v w^{1-s} w^{-2 r-1} \tilde{\alpha}^{-1} \\
& =w^{r} \tilde{\alpha} \tilde{\beta}^{-1} v^{-1} w^{-1} v^{-1} w^{s-1} v w^{-r} \tilde{\beta} v w^{-s-2 r} \tilde{\alpha}^{-1} .
\end{aligned}
$$

If $s=1$, then $C F C^{-1} F A^{-1}=1$ and therefore $\mathcal{E} \circ \mathcal{A}\left(C F C^{-1} F A^{-1}\right)=0$. If $s=0$, then $C F C^{-1} F A^{-1}=w^{r} B^{-1} w^{-r}$ and so $\mathcal{E} \circ \mathcal{A}\left(C F C^{-1} F A^{-1}\right)=-1$.

Now, we are going to study the behavior of $\mathcal{E} \circ \mathcal{A}$ after conjugating the variable $Z=w^{m} v^{n} B^{k} v^{-n} w^{-m}$ by $\tilde{\alpha}, \tilde{\alpha}^{-1}, \tilde{\beta}, \tilde{\beta}^{-1}$ and $\tilde{c}_{0}$ in each case of $\phi_{p}(1, \eta)$, where $B=w^{-1} v^{-1} w^{-1} v$.

Proposition 6.11. If $Z=w^{m} v^{n} B^{k} v^{-n} w^{-m}$ then

$$
\begin{aligned}
\mathcal{E} \circ \mathcal{A}(Z) & =\mathcal{E} \circ \mathcal{A}\left(\tilde{\alpha} Z \tilde{\alpha}^{-1}\right)=\mathcal{E} \circ \mathcal{A}\left(\tilde{\alpha}^{-1} Z \tilde{\alpha}\right)=-\mathcal{E} \circ \mathcal{A}\left(\tilde{\beta} Z \tilde{\beta}^{-1}\right) \\
& =-\mathcal{E} \circ \mathcal{A}\left(\tilde{\beta}^{-1} Z \tilde{\beta}\right)=k .
\end{aligned}
$$

(2) In each case of $\phi_{p}(1, \eta)$ we have $\mathcal{E} \circ \mathcal{A}\left(\tilde{c}_{0} Z \tilde{c}_{0}^{-1}\right)=\eta k$. 
Proof. In fact, since $Z=w^{m} v^{n} B^{k} v^{-n} w^{-m}$ and since $\tilde{\alpha} B \tilde{\alpha}^{-1}=w^{-1} B w$ from the table (4.3), it follows that $\mathcal{E} \circ \mathcal{A}(Z)=k$ and $\mathcal{E} \circ \mathcal{A}\left(\tilde{\alpha} Z \tilde{\alpha}^{-1}\right)=k$. Also $\tilde{\beta} B \tilde{\beta}^{-1}=v^{-1} w B^{-1} w^{-1} v$ and so $\mathcal{E} \circ \mathcal{A}\left(\tilde{\beta} Z \tilde{\beta}^{-1}\right)=-k$.

Now $\tilde{\alpha}^{-1} B \tilde{\alpha}=w B w^{-1}$ and therefore $\mathcal{E} \circ \mathcal{A}\left(\tilde{\alpha}^{-1} Z \tilde{\alpha}\right)=k$. Also $\tilde{\beta}^{-1} B \tilde{\beta}=$ $v w B^{-1} w^{-1} v^{-1}$ and therefore $\mathcal{E} \circ \mathcal{A}\left(\tilde{\beta}^{-1} Z \tilde{\beta}\right)=-k$. Finally, according to the table (4.4), $\tilde{c}_{0} B \tilde{c}_{0}^{-1}=B^{\eta}$ and therefore $\mathcal{E} \circ \mathcal{A}\left(\tilde{c}_{0} Z \tilde{c}_{0}^{-1}\right)=\eta k$.

Corollary 6.12. If $Z=w^{m} v^{n} B^{k} v^{-n} w^{-m}, A=\tilde{\alpha} w^{r}, F=\tilde{\beta} v w^{1-s}$ and $C=w^{c_{1}}(v w)^{c_{2}} \tilde{c}_{0}$, then in each case of $\phi_{p}(1, \eta)$,

- $\mathcal{E} \circ \mathcal{A}\left(A Z A^{-1}\right)=\mathcal{E} \circ \mathcal{A}\left(F A^{-1} Z A F^{-1}\right)=-\mathcal{E} \circ \mathcal{A}\left(A^{p} F^{\eta} Z F^{-\eta} A^{-p}\right)=k$,

- $\mathcal{E} \circ \mathcal{A}\left(C Z C^{-1}\right)=\eta k$.

Proof. This is an immediate consequence of Proposition 6.11.

Now, we will apply $\mathcal{E} \circ \mathcal{A}$ to the second and the third equations of the system $(I)$. By Corollary 6.12 and Proposition 6.2 we do not obtain any information if we apply the homomorphism to the first equation.

Let

$$
\begin{aligned}
& Z_{1}=\prod_{i} w^{u_{i}} v^{v_{i}} B^{t_{1}^{i}} v^{-v_{i}} w^{-u_{i}}, \quad Z_{2}=\prod_{i} w^{m_{i}} v^{n_{i}} B^{t_{2}^{i}} v^{-n_{i}} w^{-m_{i}} \\
& Z_{3}=\prod_{i} w^{x_{i}} v^{y_{i}} B^{t_{3}^{i}} v^{-y_{i}} w^{-x_{i}} \quad \text { and } \quad t_{j}=\sum_{i} t_{j}^{i}
\end{aligned}
$$

where $t_{j}^{i}$ is the exponent of $B$ in the $i$ th factor of $Z_{j}$.

THEOREM 6.13. If we apply $\mathcal{E} \circ \mathcal{A}$ to the second equation of the system (I) we obtain $c_{2}=0$ in Cases $\mathrm{I}$ and II, and $-2 t_{1}-1+c_{2}+r=0$ in cases III and IV.

Proof. This follows from Corollary 6.12 and Propositions 6.3 and 6.6.

We will see that the conditions given by Theorem 6.13 are also sufficient to solve the problem for Cases I and II.

For the other cases we have

Theorem 6.14. In Cases III.1 and IV, i.e. $\phi_{p}(1,-1)$ where $p=0,1$ respectively, if we apply $\mathcal{E} \circ \mathcal{A}$ to the third equation of the system $(I)$ we obtain $2\left(t_{3}-t_{2}\right)-p t_{1}-c_{2}+s-1=0$.

Proof. This follows from Corollary 6.12 and Propositions 6.9 and 6.10.

In order to solve Case III.1 completely we need some further results. The strategy will now change slightly.

Let us consider Case III.I, which corresponds to $\phi_{0}(1,-1)$. We first consider the maps $f_{1}(r, 1,0,0)$ with $r$ odd, $r \geq 3$. 
Recall that from the system generated by the input data $\left(A=\alpha w^{r}, F=\right.$ $\left.\beta v, C=c_{0} ; r, 1,(0,0)\right)$ the second equation is

$$
Z_{3}\left(C Z_{1} C^{-1}\right)\left(\prod_{i=1}^{r-1} w^{i} B w^{-i}\right)\left(A Z_{3}^{-1} A^{-1}\right) Z_{1}^{-1}=1
$$

once $C A C^{-1} A^{-1}=\prod_{i=1}^{r-1} w^{i} B w^{-i}$.

For $Z_{1}$ and $Z_{3}$ as above we have

$$
\begin{aligned}
\mathcal{A}\left(C Z_{1} C^{-1}\right) & =\sum_{i}\left(-t_{1}^{i}\right) \bar{w}^{u_{i}} \bar{v}^{-v_{i}}, \\
\mathcal{A}\left(Z_{1}^{-1}\right) & =\sum_{i}\left(-t_{1}^{i}\right) \bar{w}^{u_{i}} \bar{v}^{v_{i}}, \\
\mathcal{A}\left(A Z_{3}^{-1} A^{-1}\right) & =\sum_{i}\left(-t_{3}^{i}\right) \bar{w}^{r+x_{i}-(-1)^{y_{i}}} \bar{v}^{y_{i}}, \\
\mathcal{A}\left(Z_{3}\right) & =\sum_{i} t_{3}^{i} \bar{w}^{x_{i}} \bar{v}^{y_{i}}, \\
\mathcal{A}\left(C A C^{-1} A^{-1}\right) & =\sum_{i=1}^{r-1} 1 \bar{w}^{i} .
\end{aligned}
$$

We claim that the second equation does not have a solution.

Proposition 6.15. For Case III.1 with $\phi_{0}(1,-1)$ and $f_{1}(r, 1,0,0)$ with $r \geq 3$, the second equation of the system,

$$
Z_{3}\left(C Z_{1} C^{-1}\right)\left(C A C^{-1} A^{-1}\right)\left(A Z_{3}^{-1} A^{-1}\right) Z_{1}^{-1}=1,
$$

has no solution where $A=\alpha w^{r}$ and $C=c_{0}$.

Proof. The second equation is

$$
Z_{3}\left(C Z_{1} C^{-1}\right)\left(\prod_{i=1}^{r-1} w^{i} B w^{-i}\right)\left(A Z_{3}^{-1} A^{-1}\right) Z_{1}^{-1}=1
$$

and by taking $\mathcal{A}$ (second equation $)=0$ we get

$$
\mathcal{A}\left(Z_{3}\right)-w^{r} \mathcal{A}\left(Z_{3}\right) w^{-1}-2 \mathcal{A}\left(Z_{1}\right)+\sum_{i=1}^{r-1} 1 \bar{w}^{i}=0 .
$$

Let $H \subset \mathbb{Z}\left[\pi_{1}(K)\right]$ be the subring generated by the homogeneous elements of the form $\bar{w}^{i}$ for $i \in \mathbb{Z}$. As a group, $H$ is isomorphic to the free abelian group generated by $\bar{w}^{i}$ with $i \in \mathbb{Z}$. It is easy to see that $H$ is a subring which can be identified with the ring of Laurent polynomials over the integers. If we project the equation above on the group $H$ (the projection maps $\bar{w}^{u_{i}} \bar{v}^{v_{i}}$ to 0 if $v_{i} \neq 0$ and to $\bar{w}^{u_{i}}$ otherwise), and if by abuse of notation we write $Z_{i}$ 
for the projection of $Z_{i}$ to $H$, we get

$$
\mathcal{A}\left(Z_{3}\right)\left(1-\bar{w}^{r-1}\right)-2 \mathcal{A}\left(Z_{1}\right)+\sum_{i=1}^{r-1} 1 \bar{w}^{i}=0,
$$

which is equivalent to

$\mathcal{A}\left(Z_{3}\right)(1-\bar{w})\left(1+\bar{w}+\cdots+\bar{w}^{r-2}\right)-2 \mathcal{A}\left(Z_{1}\right)+\bar{w}\left(1+\bar{w}+\cdots+\bar{w}^{r-2}\right)=0$.

Now let us look at this equation on $H / 2 H$. Then we obtain

$$
\left(\mathcal{A}\left(Z_{3}\right)(1-\bar{w})+\bar{w}\right)\left(1+\bar{w}+\cdots+\bar{w}^{r-2}\right)=0 .
$$

This is an equation in the ring $H / 2 H$ and this ring has no zero divisors by Theorem 1.4 in [KLM88]. Therefore we must have

$$
\mathcal{A}\left(Z_{3}\right)(1-\bar{w})+\bar{w}=0, \quad \text { or } \quad \mathcal{A}\left(Z_{3}\right)(\bar{w}-1)=\bar{w}
$$

since $r \geq 3$. But the last equation has no solution (set $\bar{w}=1$ ). Hence the result follows.

Now we will consider the maps $f_{1}(r, 1,0,2)$ with $r$ odd, $r \geq 1$. This will complete the analysis of Case III.1.

We have $A=\alpha w^{r}, F=\beta v$ and $C=(v w)^{2} c_{0}$. In order to study the second equation we need the following calculation:

$$
\begin{aligned}
C A C^{-1} A^{-1}= & {\left[(v w)^{2} B(v w)^{-2}\right]\left(v^{2} B^{-2} v^{-2}\right)\left(v w^{1-r} v B v^{-1} w^{r-1} v^{-1}\right) } \\
& \cdot \prod_{i=0}^{r} v w^{i-r} B w^{r-i} v^{-1}
\end{aligned}
$$

and therefore

$$
\mathcal{A}\left(C A C^{-1} A^{-1}\right)=1 \bar{v}^{2}-2 \bar{v}^{2}+1 \bar{w}^{r-1} \bar{v}^{2}+\sum_{i=0}^{r} 1 \bar{w}^{r-i} \bar{v} .
$$

Moreover,

$$
\mathcal{A}\left(C Z_{1} C^{-1}\right)=\sum_{i}\left(-t_{1}^{i}\right) \bar{w}^{u_{i}} \bar{v}^{2-v_{i}} .
$$

We claim:

Proposition 6.16. For Case III.1 with $\phi_{0}(1,-1)$ and $f_{1}(r, 1,0,2)$ with $r \geq 0$, the second equation of the system,

$$
Z_{3}\left(C Z_{1} C^{-1}\right)\left(C A C^{-1} A^{-1}\right)\left(A Z_{3}^{-1} A^{-1}\right) Z_{1}^{-1}=1,
$$

has no solution.

Proof. As before we consider $\mathcal{A}$ (second equation $)=0$. The summands $\mathcal{A}\left(Z_{3}\right)$ and $\mathcal{A}\left(A Z_{3}^{-1} A^{-1}\right)$ are as stated before Proposition 6.15. The summands $\mathcal{A}\left(C Z_{1} C^{-1}\right)$ and $\mathcal{A}\left(C A C^{-1} A^{-1}\right)$ are calculated above. So we deduce 
that

$$
\begin{aligned}
& \sum_{i} t_{3}^{i} \bar{w}^{x_{i}} \bar{v}^{y_{i}}+\sum_{i}\left(-t_{1}^{i}\right) \bar{w}^{u_{i}} \bar{v}^{2-v_{i}}+(1-\bar{w})\left(1+\bar{w}+\cdots+\bar{w}^{r-2}\right)\left(-\bar{v}^{2}\right)+\sum_{i=0}^{r} 1 \bar{w}^{r-i} \bar{v} \\
& +\sum_{i}\left(-t_{3}^{i}\right) \bar{w}^{r+x_{i}-(-1)^{y_{i}}} \bar{v}^{y_{i}}+\sum_{i}\left(-t_{1}^{i}\right) \bar{w}^{u_{i}} \bar{v}^{v_{i}}=0,
\end{aligned}
$$

where the indices run over the $i$ 's such that $v_{i}=y_{i}=1$.

A homogeneous summand of $Z_{3}$ and its conjugate which appear in the equation are indexed by elements of the same horizontal line. A homogeneous summand of $Z_{1}$ and its conjugate which appear in the equation are indexed by elements of two horizontal lines symmetric to the line $\bar{w}=1$. In particular, if a summand is indexed by an element which belongs to the line $\bar{w}=1$ its conjugate is also a summand which is indexed by an element which belongs to the line $\bar{w}=1$. So we let $H$ be the abelian group generated by the elements $\bar{w}^{i} \bar{v}$ with $i \in \mathbb{Z}$ and we project the equation on $H$. Then we obtain the equation

$$
\left(1-\bar{w}^{r+1}\right)\left(\sum_{i} t_{3}^{i} \bar{w}^{x_{i}}\right)+2 \sum_{i}\left(-t_{1}^{i}\right) \bar{w}^{u_{i}}+\sum_{i=0}^{r} 1 \bar{w}^{r-i}=0,
$$

where the last equality follows from the fact that $\mathbb{Z}\left[\pi_{1}(K)\right]$ has no zero divisors (see Theorem 1.4 in [KLM88]).

Now we consider this equation $\bmod 2 H$, and using the fact that the group ring $\mathbb{Z}_{2}[\mathbb{Z}]$ has no zero divisors (Theorem 1.4 in [KLM88]) we obtain

$$
\left(\sum_{i} t_{3}^{i} \bar{w}^{x_{i}}\right)(1-\bar{w})\left(\sum_{i=0}^{r} 1 \bar{w}^{r-i}\right)+\sum_{i=0}^{r} 1 \bar{w}^{r-i}=0 .
$$

But this implies that

$$
\left(\sum_{i} t_{3}^{i} \bar{w}^{x_{i}}\right)(1-\bar{w})+1=0,
$$

which, as we have already seen in the proof of Proposition 6.15, does not have a solution. So the result follows.

6.2. The classification theorem. Let us consider first the cases:

I.1. $\phi_{0}(1,1)$ and $f_{s}(r, 1,0,2 k), r \geq 0, s \in\{0,1\}$ and $k \in \mathbb{Z}$,

I.2. $\phi_{0}(1,1)$ and $f_{s}(0,1, s, 2 k+1), s \in\{0,1\}$ and $k \in \mathbb{Z}$,

II. $\phi_{1}(1,1)$ and $f_{s}(2 r+1,1, r, 2 k), r \geq 0, s \in\{0,1\}$ and $k \in \mathbb{Z}$.

Proposition 6.17.

(i) Cases I.1 and II have no solution for $k \neq 0$ and Case I.2 has no solution for all $k \in \mathbb{Z}$.

(ii) In Case I.1 for $f_{s}(r, 1,0,0)$ and II for $f_{s}(2 r+1,1, r, 0), r \geq 0, s \in$ $\{0,1\}$ there is a lifting. 
Proof. For (i) we have $c_{2} \neq 0$ and the result follows from Theorem 6.13.

For (ii) let us first consider Case I.1. It is sufficient to prove that the system generated by the input data $\left(A=\tilde{\alpha} w^{r}, F=\tilde{\beta} v w^{1-s}, C=\tilde{c}_{0} ; 0,0,(0,1)\right)$ has trivial solution. In fact, in this case we have

$$
A F A F^{-1}=C A C^{-1} A^{-1}=C F C^{-1} F^{-1}=1 .
$$

So we have the trivial solution.

Similarly for II, if we take $A=\tilde{\alpha} w^{2 r+1}, F=\tilde{\beta} v w^{1-s}$ and $C=w^{r} \tilde{c}_{0}$ then the system generated by the data $(A, F, C ; 2 r+1, s,(r, 0))$ has trivial solution.

Now, let us consider Case III.1 with $\phi_{0}(1,-1)$ and $f_{s}(r, 1,0,2 k), s \in$ $\{0,1\}, r \geq 0$ and $k \in\{0,1\}$.

Corollary 6.18. Case III.1 has no solution for $r$ even or $s$ even.

Proof. The condition from Theorem 6.13 is not satisfied modulo 2 for $r$ even. The condition from Theorem 6.14 is not satisfied modulo 2 for $s$ even.

Corollary 6.19. In Case III. 1 if $r \geq 3$ is odd then the system generated by the input data $\left(A=\tilde{\alpha} w^{r}, F=\tilde{\beta} v, C=\tilde{c}_{0} ; r, 1,(0,0)\right)$ has no solution.

Proof. This follows from Proposition 6.15.

Corollary 6.20. In Case III.1, if $r \geq 1$ is odd then the system generated by the input data $\left(A=\tilde{\alpha} w^{r}, F=\tilde{\beta} v, C=(v w)^{2} \tilde{c}_{0} ; r, 1,(0,2)\right)$ has no solution.

Proof. This follows from Proposition 6.16.

Theorem 6.21. In Case III.1, if $s=1, r=1$ and $c_{2}=0$ then the system generated by the input data $\left(A=\tilde{\alpha} w, F=\tilde{\beta} v, C=\tilde{c}_{0} ; 1,1,(0,0)\right)$ has a solution.

Proof. In fact, using the tables (4.3) and (4.4) we obtain

$$
A F A F^{-1}=C A C^{-1} A^{-1}=C F C^{-1} F=1 .
$$

Now let us consider Case III.2 with $\phi_{0}(1,-1)$ and $f_{s}(0,1, s, 1), s \in\{0,1\}$.

TheOREM 6.22. The system generated by the input data $(A=\tilde{\alpha}, F=$ $\left.\tilde{\beta} v w^{1-s}, C=w^{s-1} v \tilde{c}_{0} ; 0, s,(s, 1)\right)$ for $s \in\{0,1\}$ has trivial solution. So there is a lifting for $f_{s}(0,1, s, 1)$ with $s \in\{0,1\}$.

Proof. In fact, we have $A F A F^{-1}=1$ (see proof of Proposition 6.2). Moreover, using the tables (4.3) and (4.4) we obtain

$$
C A C^{-1} A^{-1}=C F C^{-1} F=1 \text {. }
$$


Now let us consider Case IV with $\phi_{1}(1,-1)$ and $f_{s}(2 r+1,1, r, 0), r \in \mathbb{Z}$, $s \in\{0,1\}$.

Proposition 6.23. In Case IV, if $r-s=2 l, l \in \mathbb{Z}$ and $s \in\{0,1\}$, then the system generated by the input data $\left(A=\tilde{\alpha} w^{2 r+1}, F=\tilde{\beta} v w^{1-s}\right.$, $\left.C=w^{r} \tilde{c}_{0} ; 2 r+1, s,(r, 0)\right)$ has no solution.

Proof. In fact, in Case IV, $p=1$ and $c_{2}=0$ and therefore the hypotheses make it impossible to solve the system

$$
\left\{\begin{array}{l}
\mathcal{E} \circ \mathcal{A} \text { (second equation) } \\
\mathcal{E} \circ \mathcal{A} \text { (third equation) }
\end{array}=\left\{\begin{array}{l}
-2 t_{1}+2 r=0 \\
2\left(t_{3}-t_{2}\right)-t_{1}+s-1=0
\end{array}\right.\right.
$$

Now it is necessary to decide whether or not there is a lifting in each of the cases $f_{0}(2 r+1,1, r, 0)$ with $r$ odd, and $f_{1}(2 r+1,1, r, 0)$ with $r$ even.

Lemma 6.24. In Case IV, if $q=v^{-1} w v$ then

\begin{tabular}{|l|l|}
\cline { 1 - 2 }$\tilde{\alpha} q \tilde{\alpha}^{-1}=w^{-1} q w$ & \multicolumn{1}{|c}{} \\
\hline$\tilde{\beta}^{-1} q \tilde{\beta}=w^{-1}$ & $\tilde{\beta} q \tilde{\beta}^{-1}=v^{-1} q^{-1} v$ \\
\hline$\tilde{\beta} w \tilde{\beta}^{-1}=q^{-1}$ & $\tilde{c}_{0}^{-1} q \tilde{c}_{0}=w^{-1}$ \\
\hline$\tilde{c}_{0}^{-1} w \tilde{c}_{0}=q^{-1}$ & $\tilde{c}_{0} w \tilde{c}_{0}^{-1}=q^{-1}$ \\
\hline
\end{tabular}

Proof. All the above relations follow immediately from the tables (4.3) and (4.4).

Proposition 6.25. In Case IV of Corollary 5.4 there is a lifting for $f_{s}(2 r+1,1, r, 0)$ and $r+s=2 l+1, l \in \mathbb{Z}$ and $s \in\{0,1\}$.

Proof. Consider the system generated by the input data $\left(A=C F C^{-1} F\right.$, $\left.F=\tilde{\beta} w^{s-1} v, C=q^{1-s} w^{l+1-s} q^{s-l-1} \tilde{c}_{0} ; 4 l+3-2 s, s,(2 l+1-s, 0)\right)$, where $q=v^{-1} w v$.

It follows from Lemma 6.24 that $A F A F^{-1}=1$ and $C A C^{-1} A^{-1}=1$. Also, since $A=C F C^{-1} F$ we have $C F C^{-1} F A^{-1}=1$. Therefore the system generated by the input data above has trivial solution and so there is a lifting for $f_{s}(4 l+3-2 s, 1,2 l+1-s, 0), l \in \mathbb{Z}$ and $s \in\{0,1\}$.

The considerations above together with Remark 5.1 allow us to state the main result. Let $\phi$ be a homeomorphism of $K$, where $K$ denotes the Klein bottle, and let $M(\phi)$ be $K \times[0,1]$ with $(x, 0)$ with $(\phi(x), 1)$ identified. Then $M(\phi)$ is a fiber bundle over the circle $S^{1}$ with fiber $K$.

TheOREM 6.26. Let $f: M(\phi) \rightarrow M(\phi)$ be a fiber-preserving map over $S^{1}$. If $f$ belongs to one of the cases of Theorem 2.4 then it can be deformed to a fixed point free map $g$ by a fiberwise homotopy over $S^{1}$ if and only if $f$ belongs to the corresponding case in the table below. 


\begin{tabular}{|l|l|}
\hline $\begin{array}{l}\text { Case I } \\
\phi_{0}(1,1)\end{array}$ & $f_{s}(r, 1,0,0): \alpha \mapsto \alpha^{r}, \beta \mapsto \alpha^{s} \beta, c_{0} \mapsto c_{0}, r, s \in \mathbb{Z}$ \\
\hline Case II & $f_{s}(2 r+1,1, r, 0): \alpha \mapsto \alpha^{2 r+1}, \beta \mapsto \alpha^{s} \beta, c_{0} \mapsto \alpha^{r} c_{0}, r, s \in \mathbb{Z}$ \\
$\phi_{1}(1,1)$ & \\
\hline Case III & $f_{2 s+1}(1,1,0,4 k): \alpha \mapsto \alpha, \beta \mapsto \alpha^{2 s+1} \beta, c_{0} \mapsto \beta^{4 k} c_{0}, s, k \in \mathbb{Z}$ \\
$\phi_{0}(1,-1)$ & $f_{2 s+1}(-1,1,0,4 k+2): \alpha \mapsto \alpha^{-1}, \beta \mapsto \alpha^{2 s+1} \beta, c_{0} \mapsto \beta^{4 k+2} c_{0}, s, k \in \mathbb{Z}$ \\
& $f_{s}(0,1, s, 2 k+1): \alpha \mapsto 1, \beta \mapsto \alpha^{s} \beta, c_{0} \mapsto \alpha^{s} \beta^{2 k+1} c_{0} s, k \in \mathbb{Z}$ \\
\hline Case IV & $f_{2 s}(4 l+3,1,2 l+1,4 k): \alpha \mapsto \alpha^{4 l+3}, \beta \mapsto \alpha^{2 s} \beta, c_{0} \mapsto \alpha^{2 l+1} \beta^{4 k} c_{0}, s, k, l \in \mathbb{Z}$ \\
$\phi_{1}(1,-1)$ & $f_{2 s}(4 l+1,1,2 l, 4 k+2): \alpha \mapsto \alpha^{4 l+1}, \beta \mapsto \alpha^{2 s} \beta, c_{0} \mapsto \alpha^{2 l} \beta^{4 k+2} c_{0}, s, k, l \in \mathbb{Z}$ \\
& $f_{2 s+1}(4 l+3,1,2 l+1,4 k+2): \alpha \mapsto \alpha^{4 l+3}, \beta \mapsto \alpha^{2 s+1} \beta, c_{0} \mapsto \alpha^{2 l+1} \beta^{4 k+2} c_{0}, s, k \in \mathbb{Z}$ \\
& $f_{2 s+1}(4 l+1,1,2 l, 4 k): \alpha \mapsto \alpha^{4 l+1}, \beta \mapsto \alpha^{2 s+1} \beta, c_{0} \mapsto \alpha^{2 l} \beta^{4 k} c_{0}, s, k, l \in \mathbb{Z}$ \\
\hline
\end{tabular}

Acknowledgments. We would like to thank the referee for his suggestions which have greatly improved the presentation of this work.

\section{References}

[Ba77] H. J. Baues, Obstruction Theory, Lecture Notes in Math. 628, Springer, 1977.

[DJ93] R. Dobreńko and J. Jezierski, The coincidence Nielsen number on nonorientable manifolds, Rocky Mountain J. Math. 23 (1993), 67-85.

[Do74] A. Dold, The fixed point index of fibre-preserving maps, Invent. Math. 25 (1974), 281-297.

[FH81] E. Fadell and S. Husseini, A fixed point theory for fiber preserving maps, in: Lecture Notes in Math. 886, Springer, 1981, 49-72.

[FH82] E. Fadell and S. Husseini, The Nielsen number on surfaces, in: Topological Methods in Nonlinear Functional Analysis, Contemp. Math. 21, Amer. Math. Soc., 1982, 59-99.

[FLP79] A. Fathi, F. Laudenbach et V. Poenaru, Travaux de Thurston sur les surfaces, Astérisque 66-67 (1979).

[Go87] D. L. Gonçalves, Fixed points of $S^{1}$-fibrations, Pacific J. Math. 129 (1987), 297-306.

[GPV04] D. L. Gonçalves, D. Penteado and J. P. Vieira, Fixed points on torus fiber bundles over the circle, Fund. Math. 183 (2004), 1-38.

[GPV09] - - - - Abelianized obstruction for fixed points of fiber-preserving maps of surface bundles, Topol. Methods Nonlinear Anal., to appear.

[Ji85] B. Jiang, Fixed points and braids II, Math. Ann. 272 (1985), 249-256.

[Jo76] D. L. Johnson, Presentation of Groups, London Math. Soc. Lecture Note Ser. 22, Cambridge Univ. Press, 1976.

[KLM88] P. H. Kropholler, P. A. Linnell and J. A. Moody, Applications of a new Ktheoretic theorem to soluble group rings, Proc. Amer. Math. Soc. 104 (1988), 675-684.

[Ly50] R. C. Lyndon, Cohomology theory of groups with a single defining relation, Ann. of Math. 52 (1950), 650-665.

[MKS66] W. Magnus, A. Karrass and D. Solitar, Combinatorial Group Theory, Interscience, New York, 1966. 
[Pe88] D. Penteado, Sobre pontos fixos de aplicações entre fibrados com fibra superfície, ICMSC-USP São Carlos, 1988.

[Pe97] - Fixed points on surface fiber bundles, in: 10th Brazilian Topology Meeting, Mat. Contemp. 13 (1997), 251-267.

[S69] G. P. Scott, Braid groups and the group of homeomorphisms of a surface, Proc. Cambridge Philos. Soc. 68 (1969), 605-617.

[Sko87] R. Skora, The degree of a map between surfaces, Math. Ann. 276 (1987), 415423.

Daciberg Lima Gonçalves

Departamento de Matemática

IME - USP

Caixa Postal 66.281

CEP 05314-970, São Paulo, SP, Brazil

E-mail:dlgoncal@ime.usp.br

João Peres Vieira

Departamento de Matemática

I.G.C.E, São Paulo State University - UNESP

Caixa Postal 178

Rio Claro 13500-230, Brazil

E-mail: jpvieira@rc.unesp.br
Dirceu Penteado Departamento de Matemática Universidade Federal de São Carlos Rodovia Washington Luiz, Km 235

São Carlos 13565-905, Brazil E-mail: dirceu@dm.ufscar.br

Received 5 April 2008;

in revised form 19 November 2008 